\title{
Accuracy of gravitational waveform models for observing neutron-star-black-hole binaries in Advanced LIGO
}

\author{
Alexander H. Nitz, ${ }^{1}$ Andrew Lundgren, ${ }^{2,3,4}$ Duncan A. Brown, ${ }^{1,4,5}$ Evan Ochsner, ${ }^{6,4}$ Drew Keppel, ${ }^{2,3}$ and Ian W. Harry ${ }^{1,4}$ \\ ${ }^{1}$ Department of Physics, Syracuse University, Syracuse, New York 13244, USA \\ ${ }^{2}$ Albert-Einstein-Institut, Max-Planck-Institut für Gravitationsphysik, D-30167 Hannover, Germany \\ ${ }^{3}$ Leibniz Universität Hannover, D-30167 Hannover, Germany \\ ${ }^{4}$ Kavli Institute of Theoretical Physics, University of California, Santa Barbara, California 93106, USA \\ ${ }^{5}$ LIGO Laboratory, California Institute of Technology, Pasadena, California 91125, USA \\ ${ }^{6}$ Center for Gravitation and Cosmology, University of Wisconsin-Milwaukee, Milwaukee, Wisconsin 53211, USA
}

(Received 6 July 2013; published 26 December 2013)

Gravitational waves radiated by the coalescence of compact-object binaries containing a neutron star and a black hole are one of the most interesting sources for the ground-based gravitational-wave observatories Advanced LIGO and Advanced Virgo. Advanced LIGO will be sensitive to the inspiral of a $1.4 M_{\odot}$ neutron star into a $10 M_{\odot}$ black hole to a maximum distance of $\sim 900 \mathrm{Mpc}$. Achieving this sensitivity and extracting the physics imprinted in observed signals requires accurate modeling of the binary to construct template waveforms. In a neutron-star-black-hole binary, the black hole may have significant angular momentum (spin), which affects the phase evolution of the emitted gravitational waves. We investigate the ability of currently available post-Newtonian templates to model the gravitational waves emitted during the inspiral phase of neutron-star-black-hole binaries. We restrict to the case where the spin of the black hole is aligned with the orbital angular momentum and compare several post-Newtonian approximants. We examine restricted amplitude post-Newtonian waveforms that are accurate to third-and-a-half post-Newtonian order in the orbital dynamics and complete to secondand-a-half post-Newtonian order in the spin dynamics. We also consider post-Newtonian waveforms that include the recently derived third-and-a-half post-Newtonian order spin-orbit correction and the third post-Newtonian order spin-orbit tail correction. We compare these post-Newtonian approximants to the effective-one-body waveforms for spin-aligned binaries. For all of these waveform families, we find that there is a large disagreement between different waveform approximants, starting at low to moderate black hole spins, particularly for binaries where the spin is antialigned with the orbital angular momentum. The match between the TaylorT4 and TaylorF2 approximants is $\sim 0.8$ for a binary with $m_{\mathrm{BH}} / m_{\mathrm{NS}} \sim 4$ and $\chi_{\mathrm{BH}}=c J_{\mathrm{BH}} / G m_{\mathrm{BH}}^{2} \sim 0.4$. We show that the divergence between the gravitational waveforms begins in the early inspiral at $v \sim 0.2$ for $\chi_{\mathrm{BH}} \sim 0.4$. Post-Newtonian spin corrections beyond those currently known will be required for optimal detection searches and to measure the parameters of neutron-star-black-hole binaries. The strong dependence of the gravitational-wave signal on the spin dynamics will make it possible to extract significant astrophysical information from detected systems with Advanced LIGO and Advanced Virgo.

DOI: 10.1103/PhysRevD.88.124039

PACS numbers: 04.30.Db

\section{INTRODUCTION}

Compact object binaries are likely to be the first source detected by the Advanced Laser Interferometer Gravitational-wave Observatory (aLIGO) [1] and Advanced Virgo (AdV) [2]. These detectors will be sensitive to the gravitational waves radiated as the orbital frequency of the binary sweeps upwards from $\sim 5-10 \mathrm{~Hz}$ to the point at which the compact objects coalesce [3]. Binaries containing a (NSBH) have a predicted coalescence rate of $0.2-300 \mathrm{yr}^{-1}$ within the sensitive volume of aLIGO [4], making them an important source for these observatories. The observation of a NSBH by aLIGO would be the first conclusive detection of this class of compact-object binary. Gravitational-wave observations of NSBH binaries will allow us to explore the central engine of short, hard gamma-ray bursts, shed light on models of stellar evolution and core collapse, and investigate the dynamics of compact objects in the strong-field regime [5-11]. Achieving aLIGO's optimal sensitivity to NSBH binaries and exploring their physics requires accurate modeling of the gravitational waves emitted over many hundreds of orbits as the signal sweeps through the detector's sensitive band. For binary neutron star (BNS) systems the mass ratio between the two neutron stars is small and the angular momenta of the neutron stars (the neutron stars' spins) is low. In this case, the emitted waves are well modeled by post-Newtonian (PN) theory [12-14]. However, NSBH binaries can have significantly larger mass ratios and the spin of the black hole can be much larger than that of a neutron star. The combined effects of mass ratio and spin present challenges in constructing accurate gravitational waveform models for 
NSBH systems, compared to BNS systems. In this paper we investigate how accurately current theoretical models simulate NSBH gravitational waveforms within the sensitive frequency band of aLIGO.

Although no NSBH binaries have been directly observed, both black holes (BHs) and neutron stars (NSs) have been observed in other binary systems. Several BNS systems and neutron-star-white-dwarf systems have been observed by detecting their electromagnetic signatures. Electromagnetic observations suggest that the NS mass distribution in BNS peaks at $1.35 M_{\odot}-1.5 M_{\odot}$ with a narrow width [15], although NSs in globular clusters seem to have a considerably wider mass distribution [15]. There is also evidence that a neutron star in one system has a mass as high as $\sim 3 M_{\odot}[16]$. The dimensionless spin magnitude $\chi=\mathrm{cJ} / \mathrm{Gm}^{2}$ for NSs is constrained by possible NS equations of state to a maximum of 0.7 [17]. The fastest observed pulsar has a spin period of $1.4 \mathrm{~ms}$ [18], corresponding to a $\chi \sim 0.4$, and the most rapidly spinning observed NS in a binary, J0737-3039A, has a spin of only $\chi \sim 0.05$. The observational data for $\mathrm{BHs}$ is more limited than for NSs. Studies of BHs in low-mass x-ray binaries suggest a mass distribution of $7.8 \pm 1.2 M_{\odot}$ [19]. This extends to $8-11 \pm 2-4 M_{\odot}$ when five high-mass, wind-fed, x-ray binary systems are included [20]. For BHs there is evidence for a broad distribution of spin magnitudes [21], although general relativity limits it to be $\chi<1$. Given the uncertainties in the masses and spins of NSBH binaries, we consider a fairly broad mass and spin distribution when investigating the accuracy of NSBH waveforms. In this paper, we consider NSBH binaries with the NS mass between 1 and $3 M_{\odot}$, the BH mass between 3 and $15 M_{\odot}$, the NS spin between 0 and 0.05 and the $\mathrm{BH}$ spin between 0 and 1 . Between these limits, the distributions of mass and spin are all assumed to be uniform.

Gravitational-wave detectors are sensitive to the phase evolution of the waves radiated by the binary. PN theory can be used to compute the energy of a compact binary $E(v)$ and the flux radiated in gravitational waves $\mathcal{F}(v)$ in terms of the invariant velocity $v=(\pi M f)^{1 / 3}$, where $M=m_{1}+m_{2}$ is the total mass of the binary, and $f$ is the gravitational-wave frequency [12]. By solving the energy balance equation $d E / d t=-\mathcal{F}$, we can obtain expressions for the gravitational-wave phase as a function of time $\phi(t)$ or, equivalently, the Fourier phase of the waves as a function of frequency $\Psi(f)$. At leading order, the gravitational-wave phase depends only on the chirp mass $\mathcal{M}_{c}=\left(m_{1} m_{2}\right)^{3 / 5} /\left(m_{1}+m_{2}\right)^{1 / 5}$ [22]. Beyond leading order, the waveforms also depend on the symmetric mass ratio $\eta=m_{1} m_{2} /\left(m_{1}+m_{2}\right)^{2}$ [23-28], with spinorbit corrections entering at the third correction beyond leading order [29-33].

There are several different ways in which to solve the energy balance equation to obtain the gravitational-wave phase measurable by aLIGO; these different methods are known as PN approximants. While the convergence of the full PN series is not guaranteed, the available PN approximants produce waveforms that are indistinguishable for BNS systems in Advanced LIGO and are reliable for use in detection searches and parameter measurement [13,14,34]. However, for NSBH binaries the total mass, and hence the PN expansion parameter $v$, is larger. The mass ratio and spin corrections are also more significant. In this paper, we investigate the accuracy of waveforms generated by different PN approximants for observing NSBH binaries with aLIGO. To do this, one could compare subsequent terms in the PN expansion and determine the effect of neglecting them. However, in the case of systems whose component objects are spinning, only terms up to $2.5 \mathrm{PN}$ order are completely known [29-31]. This represents the leading order (1.5PN) and next-to-leading order (2.5PN) spin-orbit, along with the leading order (2.0PN) spin-spin contributions to the phasing [29-31]. We choose to compare approximants that are constructed with terms up to the same PN order, but that use inversely related differential equations to solve for the orbital dynamics, in addition to comparing to approximants that include higher order spinrelated corrections at partially derived orders [33,35]. These methods both have the effect of testing how well the PN series has converged. We also present a comparison between waveforms from these PN approximants where we fix the mass and spin parameters of the objects in order to understand when in the inspiral the waveforms diverge.

We consider two families of PN approximants for binaries where the spin of the black hole is aligned with the orbital angular momentum: TaylorT2 [12,26,36] and TaylorT4 [37]. In these models, we include all the completely known orbital evolution terms (up to 3.5PN order) [23-28] and all the completely known spin-related terms (up to 2.5PN order) [29,38-41]. Restricting to systems where the spin angular momenta are aligned (or antialigned) with the orbital angular momentum means that the plane of the binary does not precess, simplifying our comparisons. However, this study captures the dominant effect of spin on the waveforms [42]. In a separate paper, we investigate the effect of precession on detection searches [43]. We also consider the effective-one-body model as described in Ref. [44]. We separately consider models that include spin-related terms up to 3.5PN order [33,35]. Spin-orbit tail (3.0PN) and next-to-next-to-leading order spin-orbit (3.5PN) contributions to the phasing are known. However, these orders are incomplete as there are also unknown spin corrections at 3.0PN and 3.5PN, including spin-spin and (spin-induced) octupole-monopole couplings.

We restrict to comparing the inspiral portion of approximants. Numerically modelling the merger of a black hole and a neutron star is an active area of research [45-49]. However, producing long simulations of NSBH systems with high spin remains a challenge, and there is currently 


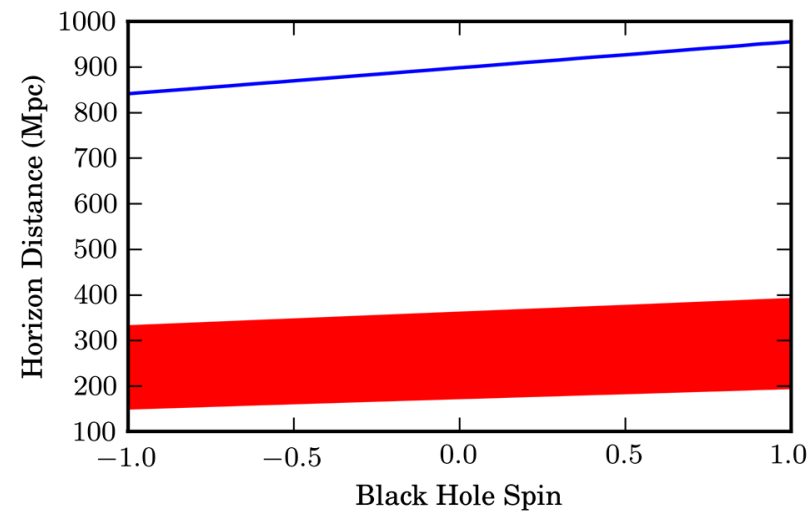

FIG. 1 (color online). The horizon distance as a function of the spin of the black hole for a $1.4 M_{\odot}-10 M_{\odot} \mathrm{NSBH}$ system, for both the aLIGO zero-detuned, high-power aLIGO sensitivity curve (blue) and plausible early aLIGO detector sensitivities (red), with a $15 \mathrm{~Hz}$ lower frequency cutoff. Results are obtained using the TaylorT4 approximant including only the complete spin terms up to 2.5PN. Note that aLIGO will be sensitive to NSBH systems out to $\sim 900 \mathrm{Mpc}$, and there will be increased sensitivity for systems with aligned black hole spins with large magnitudes.

no widely available waveform model that includes the complete evolution of a NSBH coalescence over the full parameter space we consider. Refs. [13,50] suggest that for nonspinning systems, inspiral-only templates are suitable for detection purposes below a total mass of $12 M_{\odot}$. For a canonical $1.4 M_{\odot}$ neutron star, this roughly corresponds to a mass ratio of 8 . Even at the upper range of masses we consider, $(3+15) M_{\odot}$, it has been shown in the case of nonspinning numerically modeled binary black hole waveforms that inspiral-only template banks recover $>95 \%$ of the signal power [50,51].

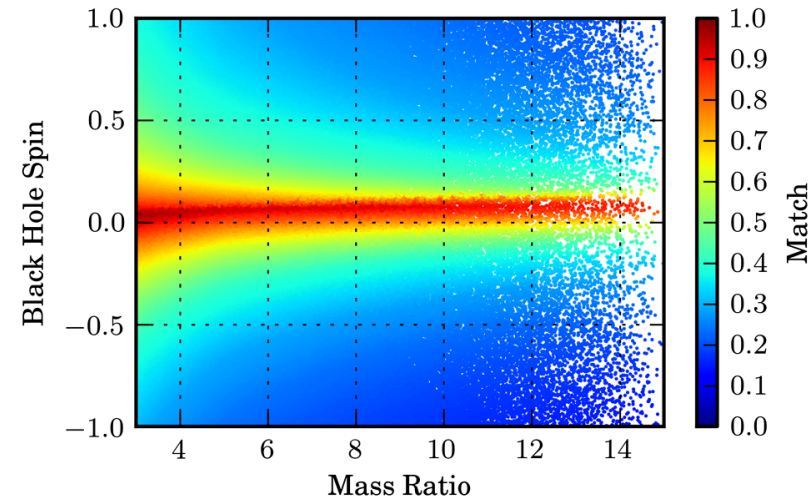

FIG. 2 (color online). The match between the TaylorF2 and TaylorT4 approximants as a function of the spin of the black hole and the mass ratio of the system. Only the completely known spin-related corrections up to $2.5 \mathrm{PN}$ are included. Matches are calculated using the aLIGO zero-detuned, high-power sensitivity curve and a $15 \mathrm{~Hz}$ lower frequency cutoff. A significant reduction in match is seen for even moderate spins $\chi \sim 0.3$ and low mass ratios $m_{b h} / m_{n s} \sim 4$. The approximants also begin to disagree for nonspinning systems as the mass ratio increases.

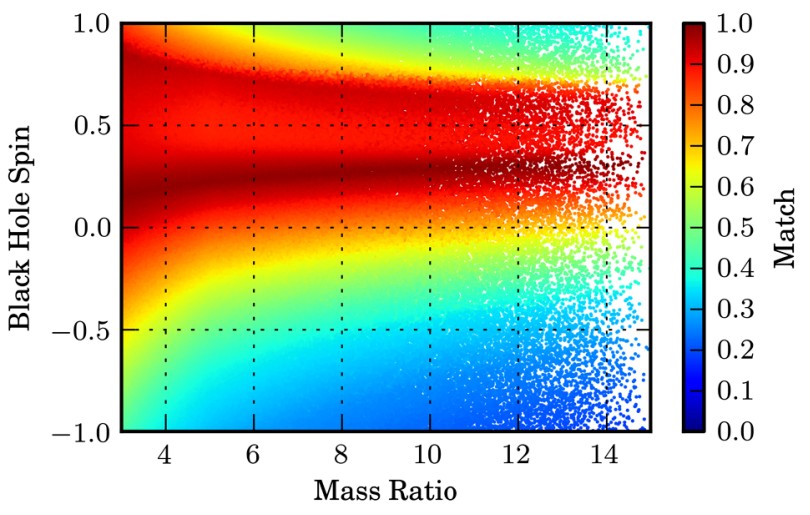

FIG. 3 (color online). The match between the TaylorF2 and TaylorT4 approximants as a function of black hole spin and mass ratio. Both models include the next-to-next-to-leading spin-orbit (3.5PN) and spin-orbit tail terms (3.0PN). In comparison to Fig. 2, the additional terms have improved the agreement for moderately spinning aligned spin systems; however, the match is still $\sim 0.8$ for $\chi \sim 0.5$ at all mass ratios.

In Fig. 1 we show the distance an optimally oriented system would be observed at signal-to-noise ratio (SNR) 8 (the horizon distance), for a $1.4 M_{\odot}-10 M_{\odot}$ NSBH system, as a function of the spin of the black hole, for both the aLIGO zero-detuned, high-power sensitivity curve and a plausible range of early aLIGO sensitivities [52]. Systems where the spin of the black hole is large in magnitude and aligned with the orbital angular momentum can be seen from a greater distance than systems where the spin is small or antialigned. Achieving this sensitivity requires NSBH waveforms that do not incur a significant loss in SNR when used as search templates [53]. Furthermore,

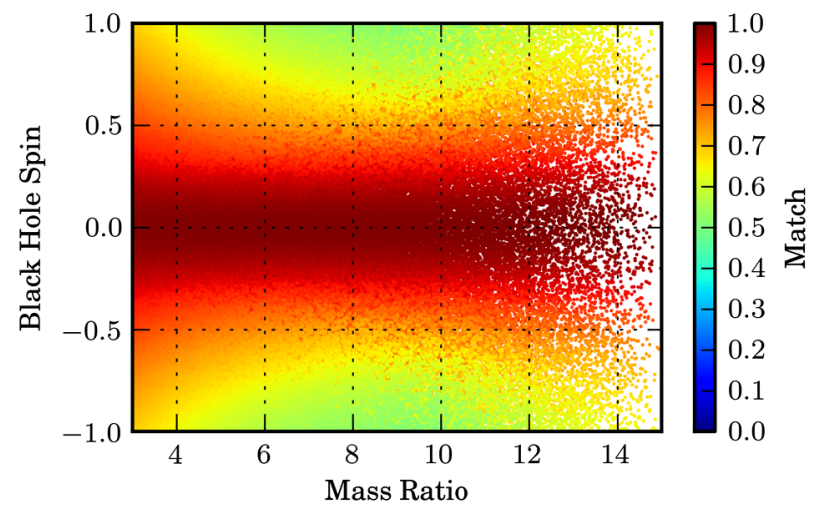

FIG. 4 (color online). The match between TaylorF2 with 2.5PN spin corrections and TaylorF2 including the next-tonext-to-leading spin-orbit (3.5PN) and spin-orbit tail terms (3.0PN), as a function of the spin of the black hole and the mass ratio of the system. Matches are calculated using the aLIGO zero-detuned, high-power sensitivity curve and a $15 \mathrm{~Hz}$ lower frequency cutoff. Although there is agreement where the spins are low $\chi<0.2$, the match quickly drops as the spin of the black hole increases, so that the match is already $\sim 0.7$ for $\chi \sim 0.5$. 


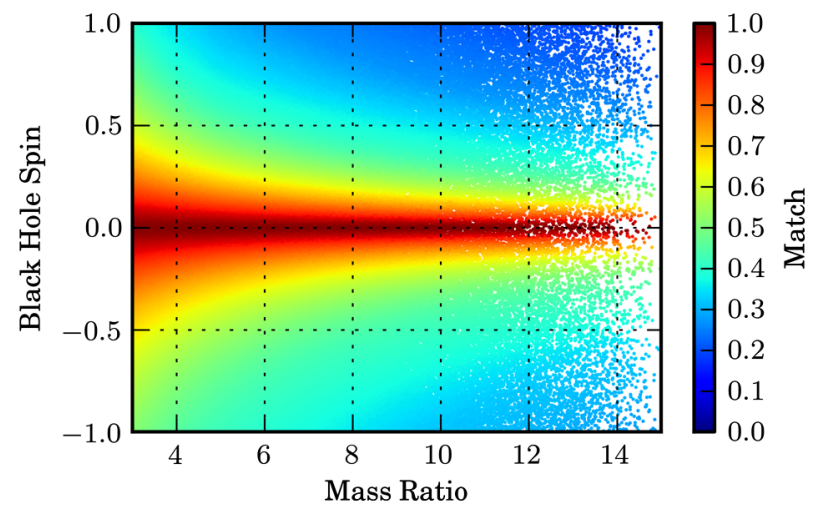

FIG. 5 (color online). The match between TaylorT4 with 2.5PN spin corrections and TaylorT4 including the next-tonext-to-leading spin-orbit (3.5PN) and spin-orbit tail terms (3.0PN), as a function of the spin of the black hole and the mass ratio of the system. Matches are calculated using the aLIGO zero-detuned, high-power sensitivity curve and a $15 \mathrm{~Hz}$ lower frequency cutoff. In comparison to Fig. 4, the approximant is more noticeably changed by the additional terms. For a mass ratio of 8 , the match has already fallen to $\sim 0.7$ for $\chi \sim 0.15$.

extracting the physics from observed signals requires faithful templates for parameter measurement.

We find that no presently available waveform model is sufficiently accurate for use in parameter measurement. Our key results, Figs. 2-6, show the match between the various waveform families considered here. There is a significant disagreement between the PN approximants we have examined, even at low $(\chi \sim 0.4)$ spins and small $\left(m_{\mathrm{BH}} / m_{\mathrm{NS}} \sim 4\right)$ mass ratios for TaylorF2 and TaylorT4. The match decreases as these increase with matches as low as $\sim 0.1$ observed. This motivates the need to compute higher order PN spin corrections.

Our present knowledge of NSBH waveforms will limit the ability of gravitational-wave observatories to accurately determine source parameters from the detected signals and may hinder the detection of some sources. Further analytical and numerical modeling of NSBH systems will be needed before aLIGO comes online in 2015 and reaches full sensitivity in $\sim 2019$ [52].

The remainder of this paper is organized as follows. In Sec. II, we describe the construction of the PN approximants used and Sec. III describes our method of comparing them. In Sec. IV we show the results of comparing different $\mathrm{PN}$ approximants, and show that there is a large discrepancy between the waveforms for NSBH binaries at relatively low black hole spins. In Sec. V we construct a new frequency domain approximant that is designed to agree with TaylorT4. This is followed by a comparison of the time domain approximants to their frequency domain counterparts in Sec. VI, where we demonstrate that they largely agree. Finally, in Sec. VII and VIII we investigate where in the inspiral the disagreement between the waveform families becomes important. We demonstrate that the divergence occurs at surprisingly low velocities for even modest black hole spins. Finally in Sec. IX we investigate whether maximizing over the mass and spin parameters of the waveform can improve the agreement between present models, and investigate the accuracy of the waveforms for early aLIGO observations when the detectors will have reduced low-frequency sensitivity when compared to the ultimate sensitivity.

\section{CONSTRUCTING POST-NEWTONIAN WAVEFORMS}

We examine the accuracy and convergence of currently known waveforms for NSBH binaries by comparing approximants constructed using the PN approximations of the binary's equation of motion and gravitational radiation. To obtain the gravitational-wave phase from these quantities, we assume that the binary evolves adiabatically through a series of quasi-circular orbits. This is a
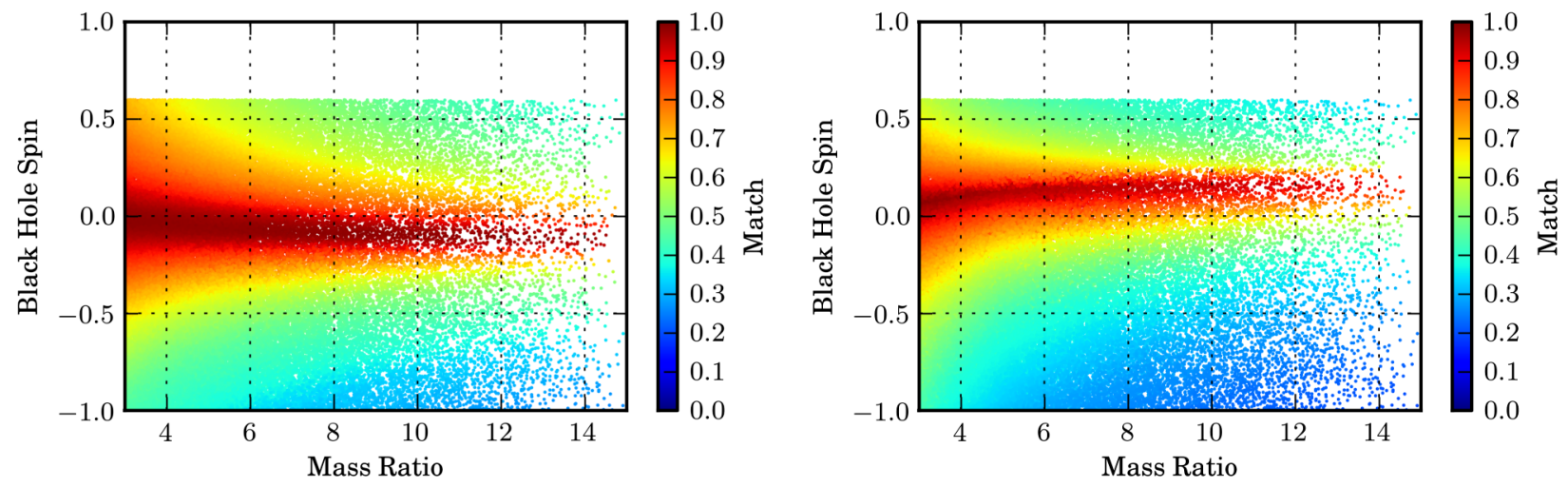

FIG. 6 (color online). The match between the TaylorF2 (left) or TaylorT4 (right) and SEOBNRv1 approximants. Spin corrections for the PN approximants are included up to 2.5PN. Matches are calculated using the aLIGO zero-detuned, high-power sensitivity curve with a $15 \mathrm{~Hz}$ lower frequency cutoff. As in Fig. 2, there is a significant reduction in match where spin of the black hole is only moderate. Note, however, that the PN approximants have marginally better agreement with SEOBNRv1 than with each other. 
reasonable approximation as gravitational radiation is expected to circularize the orbits of isolated binaries [54]. In this limit, the equations of motion reduce to series expansions of the center-of-mass energy $E(v)$ and gravitationalwave flux $\mathcal{F}(v)$, which are expanded as a power series in the orbital velocity $v$ around $v=0$. They are given as

$$
\begin{gathered}
E(v)=E_{\mathrm{N}} v^{2}\left(1+\sum_{n=2}^{6} E_{i} v^{i}\right), \\
F(v)=F_{\mathrm{N}} v^{10}\left(1+\sum_{n=2}^{7} F_{i} v^{i}\right),
\end{gathered}
$$

where the coefficients $\left\{E_{\mathrm{N}}, E_{i}, F_{\mathrm{N}}, F_{i}\right\}$ are defined in Appendix A. For terms not involving the spin of the objects, the energy is known to order $v^{6}$, while the flux is known to $v^{7}$, referred to as 3.0 and 3.5PN, respectively. At order 3.0PN, the flux contains terms proportional to both $v^{6}$ and $v^{6} \log v$; which are regarded to be of the same order. Complete terms involving the spins of the objects first appear as spin-orbit couplings at $1.5 \mathrm{PN}$ order, with spinspin couplings entering at $2 \mathrm{PN}$ order, and next-to-leading order spin-orbit couplings known at 2.5PN order.

We use the assumption that these systems are evolving independently to relate the PN energy and gravitationalwave flux equations, i.e. the loss of energy of the system is given by the gravitational-wave flux

$$
\frac{d E}{d t}=-\mathcal{F} .
$$

This can be rearranged to give an expression for the time evolution of the orbital velocity,

$$
\frac{d v}{d t}=-\frac{\mathcal{F}(v)}{E^{\prime}(v)},
$$

where $E^{\prime}(v)=d E / d v$. The orbital evolution can be transformed to the gravitational waveform by matching the near-zone gravitational potentials to the wave zone. The amplitude of gravitational waves approximated in this way are given by the PN expansion of the amplitude. This gives different amplitudes for different modes of the orbital frequency. The dominant gravitational-wave frequency $f$ is given by twice the orbital frequency, which is related to the orbital velocity by $v=(\pi M f)^{1 / 3}$. The orbital phase is therefore given by

$$
\frac{d \phi}{d t}=\frac{v^{3}}{M}
$$

and the phase of the dominant gravitational-wave mode is twice the orbital phase. Here, we will only expand the gravitational-wave amplitude to Newtonian order (0PN), which, when combined with the phase, is referred to as a restricted $\mathrm{PN}$ waveform.

Solutions $v(t)$ and $\phi(t)$ to Eqs. (4) and (5) can be used to construct the plus and cross polarizations and the observed gravitational waveform. For restricted waveforms, these are

$$
\begin{gathered}
h_{+}(t)=-\frac{2 M \eta}{D_{L}} v^{2}\left(1+\cos ^{2} \theta\right) \cos 2 \phi(t), \\
h_{\times}(t)=-\frac{2 M \eta}{D_{L}} v^{2} 2 \cos \theta \sin 2 \phi(t), \\
h(t)=F_{+} h_{+}(t)+F_{\times} h_{\times}(t) .
\end{gathered}
$$

Here $F_{+}$and $F_{\times}$are the antenna pattern functions of the detector, $D_{L}$ is the luminosity distance between the binary and observer, and $\theta$ is the inclination angle between the orbital angular momentum of the binary and the direction of gravitational-wave propagation: $\cos \theta=\hat{L} \cdot \hat{N}$. Thus, a nonprecessing, restricted PN waveform is fully specified by $v(t)$ and $\phi(t)$ [or equivalently $t(v)$ and $\phi(v)$ ].

We now have the ingredients necessary to produce the TaylorT2 and TaylorT4 families of approximants, which we describe in the following sections.

\section{A. TaylorT4}

The TaylorT4 approximant, introduced in [37], is formed by numerically solving the differential equation

$$
\frac{d v}{d t}=\left[\frac{-\mathcal{F}(v)}{E^{\prime}(v)}\right]_{k}=A_{k}(v) .
$$

The notation $[Q]_{k}$ indicates that the quantity $Q$ is to be truncated at $v^{k}$ order. Terms containing pieces logarithmic in $v$ are considered to contribute at the order given by the nonlogarithmic part. Thus waveforms expanded to 3.5PN order in the phase would be truncated at $k=7$. We use $A_{k}$ as shorthand for the truncated quantity that is used as the expression for $d v / d t$.

The resulting differential equation, given explicitly in Appendix B 1, is nonlinear and therefore must be solved numerically. The result is a function $v(t)$. The phase can then be calculated by

$$
\frac{d \phi}{d t}=\frac{v(t)^{3}}{M} .
$$

The phase is integrated from a fiducial starting frequency up to the minimum energy circular orbit (MECO), which is defined by

$$
\frac{d E(v)}{d v}=0 .
$$

The MECO frequency is where we consider the adiabatic approximation to have broken down. Note that the MECO frequency is dependent on not only the masses but also the spins of the objects; specifically, systems where the objects' spins are aligned with the orbital angular momentum will have a higher MECO frequecy. When the partial spinrelated terms at 3.0PN and 3.5PN are included, however, 
there are regions of the NSBH parameter space for which the MECO condition is never satisfied. For these cases, we impose that the rate of increase in frequency must not decrease (i.e. we stop if $d v / d t \leq 0$ ), and that the characteristic velocity of the binary is less than $c$ (i.e. we stop if $v \geq 1$ ). We terminate the waveforms as soon as any of these stopping conditions are met.

\section{B. TaylorT2}

In contrast to the TaylorT4 approximant, the TaylorT2 approximant is constructed by expanding $t$ in terms of $v$ and truncating the expression to consistent PN order. We first construct the quantity

$$
\frac{d t}{d v}=\left[\frac{E^{\prime}(v)}{-\mathcal{F}(v)}\right]_{k}=B_{k}(v) .
$$

This can be combined with the integral of (5) and solved in closed form as a perturbative expansion in $v$,

$$
\phi(v)=\int \frac{v^{3}}{M} B_{k}(v) d v .
$$

The explicit result of this integral is given in Appendix B 2 . Similar to TaylorT4, the phase is generally calculated up to the MECO frequency. However, for some points of parameter space, this formulation can result in a frequency that is not monotonic below the MECO frequency. As with TaylorT4, we stop the waveform evolution with $d v / d t \leq 0$ or $v \geq 1$.

A related approximant can be computed directly in the frequency domain by using the stationary phase approximation [12,36]. This approximant is called TaylorF2 and can be expressed as an analytic expression of the form

$$
\phi(f)=A(f) e^{i \psi(f)},
$$

where the phase takes the form

$$
\psi(f)=\sum_{i=0}^{7} \sum_{j=0}^{1} \lambda_{i, j} f^{(i-5) / 3} \log { }^{j} f .
$$

The full expressions for the amplitude and phase are given in Appendix B 3. Because the stationary phase approximation is generally valid, the TaylorT2 and TaylorF2 approximants are nearly indistinguishable [36]. An advantage of the TaylorF2 approximant comes from the fact that it can be analytically calculated in the frequency domain. In practice, waveforms that are generated in the frequency domain without the use of integration are less computationally costly, and so searches for gravitational waves from inspiraling binary systems have been performed using the TaylorF2 approximant [12,26,36,55-66].

\section{SEOBNRv1}

An additional approximant we use is the spinning effective one-body model (SEOBNRv1), presented in Ref. [44]. This approximant incorporates the results of black hole perturbation theory, the self-force formalism and PN results. The model has been calibrated to numerical relativity simulations, including simulations where the objects' spins were (anti-) aligned with the orbital angular momentum and had magnitudes of $\chi \pm 0.4$. In order to compare these waveforms more fairly with the PN approximants that only model the inspiral, we truncate this model before the merger section of the waveform.

The implemented versions of SEOBNRv1 are currently limited to $\chi \leq 0.6$. To further extend the model would require better modeling of the plunge physics and possibly the computation and incorporation of additional PN terms.

\section{COMPUTING FAITHFULNESS}

Searches for gravitational waves from compact binary coalescences utilize matched filtering $[67,68]$, in which the signal model is correlated with the detector output to construct a signal-to-noise ratio. If the signal model does not accurately capture the true gravitational waveform, then the signal-to-noise ratio, and hence the distance to which the detector can see signals at a given false alarm rate, will decrease. Matched-filtering therefore relies on the accuracy of the models. We quantify the agreement between waveform families by computing the match, or faithfulness of the waveforms, defined as follows. We define the noise-weighted inner product between two gravitational waveforms, $h_{1}$ and $h_{2}$, to be

$$
\left(h_{1} \mid h_{2}\right)=4 \Re \int_{0}^{\infty} \frac{\tilde{h}_{1}(f) \tilde{h}_{2}^{*}(f)}{S_{n}(f)} d f,
$$

where

$$
\tilde{h}_{1}(f)=\int_{0}^{\infty} h_{1}(t) e^{-2 \pi i f t} d t
$$

is the Fourier transform of $h_{1}(t)$, and $S_{n}(f)$ denotes the onesided power spectral density of the gravitational-wave detector's noise. In practice, the signals are discretely sampled so the upper frequency limit is the Nyquist frequency of the data, and the lower frequency limit of the integral is set by the detector's low-frequency sensitivity [68]. We define the normalized overlap between two waveforms $h_{1}$ and $h_{2}$ as

$$
\left(h_{1} \mid h_{2}\right)=\frac{\left(h_{1} \mid h_{2}\right)}{\sqrt{\left(h_{1} \mid h_{1}\right)\left(h_{2} \mid h_{2}\right)}} .
$$

The match between two waveforms is obtained by maximizing the overlap over the phase of the waveform and $\phi_{c}$ and any time shifts $t_{c}$ between $h_{1}$ and $h_{2}$,

$$
\mathcal{M}\left(h_{1}, h_{2}\right)=\max _{\phi_{c}, t_{c}}\left(h_{1} \mid h_{2}\left(\phi_{c}, t_{c}\right)\right),
$$

where the shifted waveform can be constructed as

$$
\tilde{h}\left(\phi_{c}, t_{c}\right)=\tilde{h} e^{i\left(\phi_{c}-2 \pi f t_{c}\right)} .
$$


The faithfulness of representing a waveform from a given PN family with that of another is described by the match between the two waveforms when the same physical parameters are used as input to the models. As both models describe the same physical source, the match should be unity. Any deviation is due to the variation between models and the match gives the fractional loss in signal-to-noise ratio that will result.

\section{POST-NEWTONIAN APPROXIMANT FAITHFULNESS COMPARISON}

In this section we compare the faithfulness between waveforms from different PN approximants where we choose the physical parameters to be consistent with NSBH sources. We also consider how the waveforms from the PN approximants compare to the waveforms from the SEOBNRv1 effective-one-body model [44]. Lastly, we consider the effect of including the spin-related terms at only partially derived orders. We model the sensitivity of second generation gravitational-wave detectors with the aLIGO zero-detuned, high-power sensitivity curve [69]. For this study we use a lower frequency cutoff of $15 \mathrm{~Hz}$ since it is not expected that detectors will have significant sensitivity below this frequency. We consider the effect of increasing this low-frequency cutoff to simulate early aLIGO sensitivities in Sec. IX.

In Fig. 2, we examine the faithfulness of NSBH waveforms by computing the match between the TaylorF2 and TaylorT4 PN approximants. The TaylorT4 approximant was used to simulate NSBH binaries in LIGO's previous gravitational-wave searches, and the TaylorF2 family is used as the templates for detection [63]. In order to focus on the mismatches primarily due to phase differences between the models, the frequency cutoff of the TaylorF2 waveform is made to agree with the ending frequency of the TaylorT4 waveform. We see that the agreement between the two models is primarily influenced by the magnitude of the black hole's spin, and secondarily by the mass ratio. There is a noticeable drop in match at higher mass ratios, even when the spin of the black hole is zero. As expected, the best agreement is seen when the black hole's spin is small and the black hole and neutron star have comparable masses. However, this plot shows that there is a substantial disagreement between these approximants for even moderately low black hole spins $(\chi \sim 0.3)$, which increases as the spin of the black hole increases. We note that the effect on the match due to the spin of the neutron star is negligible in all areas. In Fig. 3 we compare the TaylorF2 and TaylorT4 models, with the inclusion of the spin-orbit tail (3.0PN) and next-to-next-to-leading spin-orbit (3.5PN) corrections recently computed in Refs. [32,70]. In comparison to Fig. 2, the agreement is significantly improved for aligned spins with moderate magnitudes. However, these approximants maintain a poor level of overall agreement, with matches of only $\sim 0.8$ at $\chi \sim 0.5$ for all mass ratios, and even lower matches for antialigned systems. Figures 4 and 5 compare the TaylorT2 and TaylorT4 approximants with and without these additional spin terms. We see that TaylorT4 is especially sensitive to the additional corrections. In both cases, however, we note that the additional terms have caused a significant change in the waveforms, as indicated by the low matches, demonstrating that the expansion has not yet sufficiently converged to produce reliable waveforms for parameter estimation.

In Fig. 6 we compare the SEOBNRv1 model to the PN models TaylorF2 and TaylorT4. Since the SEOBNRv1 model is not valid for large values of $\chi$ [44] we restrict $\chi<$ 0.6 and only report matches below this limit. We see that, similar to the comparison between TaylorF2 and TaylorT4, these models also have large mismatches when the spin of the black hole is nonzero. The large discrepancy between the waveform families indicates that higher order PN correction terms are required. This may also pose significant problems for parameter estimation of NSBH sources.

\section{THE TAYLORR2F4 APPROXIMANT}

In the previous section, we found a surprisingly large disagreement between the TaylorF2 and TaylorT4 PN approximants when compared with waveform parameters appropriate for NSBH systems. We would like to distinguish how much of this is due to differences between time domain and frequency domain approximants, and how much of this is due to differences between the formulation of the two PN families. This can easily be performed for the TaylorF2 and TaylorT2 approximants, however we need to construct an equivalent frequency domain version of TaylorT4 to complete the comparison.

By analogy with TaylorF1 and TaylorF2 [13,71], TaylorF4 is obtained by numerically integrating the reciprocal of Eq. (9) in the frequency domain,

$$
d t / d v=1 / A_{k}(v) .
$$

However, this does not elucidate the differences between the TaylorT4 and TaylorF2 approximants. Instead, we construct an analytical approximation to the TaylorF4 approximant, which we call TaylorR2F4, by expanding Eq. (21) in powers of $v$. In order to make this series finite, we truncate these additional terms at an order in $v$ higher than the order where the PN expansion of the energy and flux were truncated,

$$
\frac{d t}{d v}=\left[\frac{1}{A_{k}(v)}\right]_{l}=B_{k}(v)+R_{k l}(v)=C_{k l}(v) .
$$

Here $B_{k}(v)$ is the same as in the TaylorT2 approximant and $R_{k l}(v)$ are the terms from order $v^{k+1}$ up to order $v^{l}$. It is important to note that this produces a power series that is identical to the TaylorF2 approximant up to the point where (12) was truncated. Thus, terms of higher order in 
$v$ account for the differences between the TaylorT2 and TaylorT4 approximants.

In Sec. VI we show that TaylorR2F4 agrees well with the TaylorT4 approximant when expanded to $v^{9}$ or $v^{12}$, which we shall see in the next section. As noted above, the second expansion in the TaylorR2F4 approximant is a different expansion than the PN expansion of the energy and flux. The Fourier phase for the TaylorR2F4 approximant can be obtained from (13) where $B_{k}(v)$ is replaced by $C_{k l}(v)$. This is given up to order $v^{N}$ as

$$
\psi_{\mathrm{R} 2 \mathrm{~F} 4}(f)=\psi_{F 2}(f)+\sum_{i=6}^{N} \sum_{j=0}^{N} \lambda_{i, j} f^{(i-5) / 3} \log { }^{j} f,
$$

where the form of these expressions up to $N=12$ can be found in Appendix B 4. Because this approximant can be analytically expressed in the frequency domain, it can be generated relatively cheaply compared to TaylorT4. This means that it has the potential to be used where computational efficiency and a higher degree of agreement with TaylorT4 is desired. We note that the frequencydomain approximants are much faster than their timedomain counterparts, which must integrate differential equations and perform a Fourier transform. Therefore, they are especially useful in computational problems which are waveform-generation limited, such as parameter estimation of signals [72].

\section{COMPARISON OF FREQUENCY TO TIME DOMAIN APPROXIMANTS}

In this section, we investigate to what extent the discrepancy between the waveform families that was demonstrated in Sec. IV is due to the difference between expressing approximants in the frequency and time domain alone. We compare the new TaylorR2F4 approximant from Sec. V, and TaylorF2, to their time domain equivalents.

We find that TaylorF2 waveforms are a good representation of TaylorT2 waveforms, even when we consider

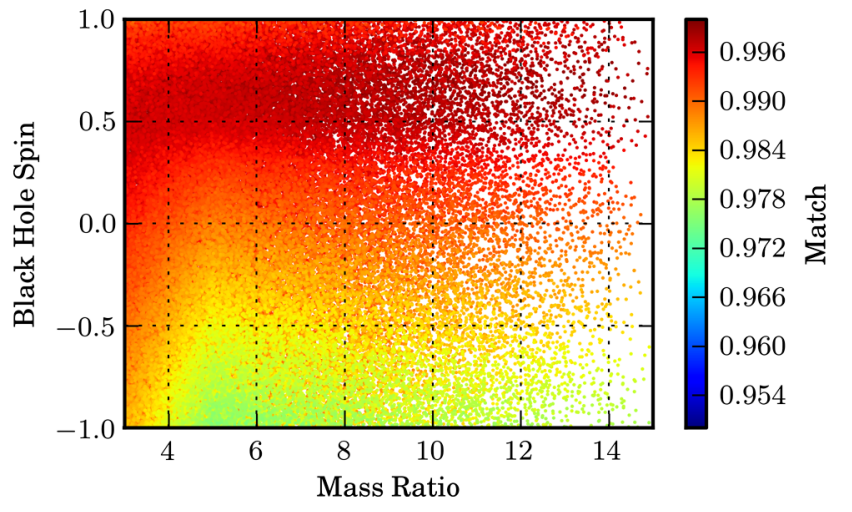

FIG. 7 (color online). The match between TaylorF2 and TaylorT2. Both include spin corrections up to 2.5PN order. Matches are calculated using the aLIGO zero-detuned, highpower sensitivity curve and a $15 \mathrm{~Hz}$ lower frequency cutoff. We see that the F2 and T2 approximants largely agree. The discrepancy between the two approixmants can be reduced by expanding the frequency sweep of the TaylorF2 approximant's amplitude to higher PN orders. However, there is different Gibbs phenomena between the two approximants that will cause a discrepancy.

waveforms from NSBH systems where the component objects are spinning. This can be seen in Fig. 7, which shows the match between the TaylorF2 and TaylorT2 models. In that figure, the ending frequency of both models is made to be the same, which is accomplished by terminating the TaylorF2 waveforms at the frequency where the generation of the equivalent TaylorT2 waveforms terminated. We find that the TaylorF2 and TaylorT2 waveforms agree to better than $\gtrsim 95.7 \%$ for the entire region investigated. For systems where the black hole spin was positively aligned with the orbital angular momentum, the match is $\gtrsim 97.9 \%$. The discrepancy between these two models is in part due to expanding to only Newtonian order the frequency sweep associated with the stationary phase approximation of the TaylorF2 approximant. In addition,
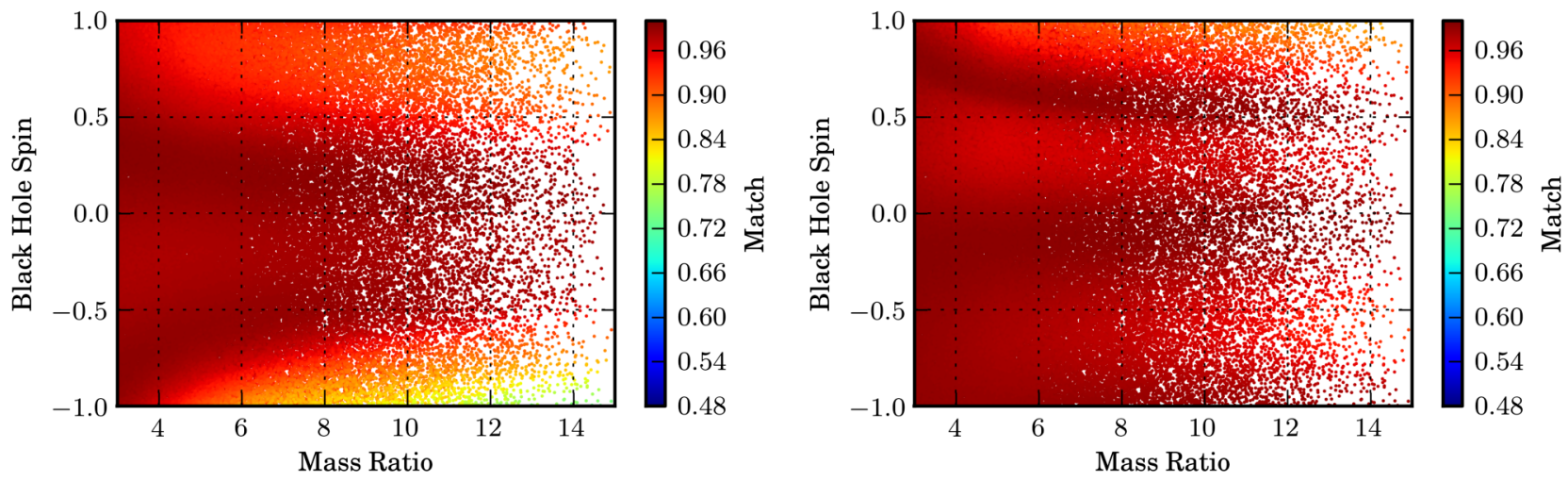

FIG. 8 (color online). The match between TaylorT4 and TaylorR2F4. Both models include spin corrections up to 2.5 PN. TaylorR2F4 is re-expanded up to order $v^{9}$ (left) and $v^{12}$ (right). Matches are calculated using the aLIGO zero-detuned, high-power sensitivity curve and a $15 \mathrm{~Hz}$ lower frequency cutoff. R2F4 and T4 have high agreement over a broad range of parameters, with some visible exceptions. Expanding up to order $v^{12}$ has generally increased agreement with TaylorT4. 


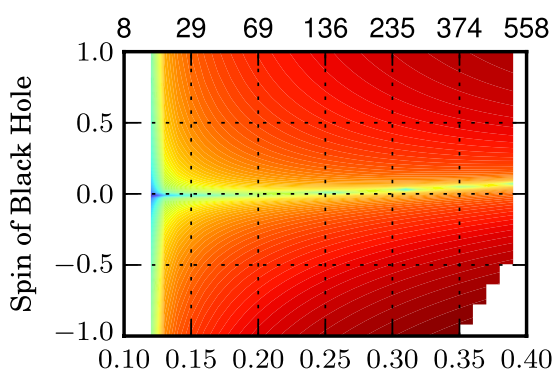

$\begin{array}{lllllll}0.10 & 0.15 & 0.20 & 0.25 & 0.30 & 0.35 & 0.40\end{array}$

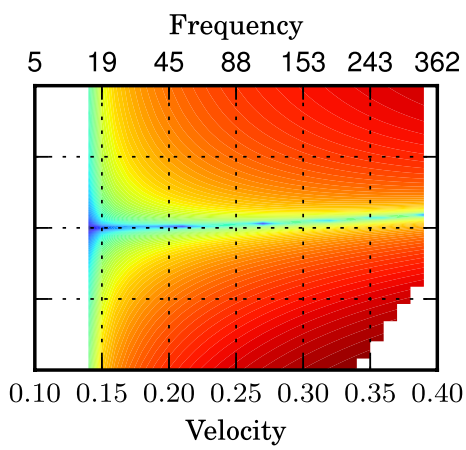

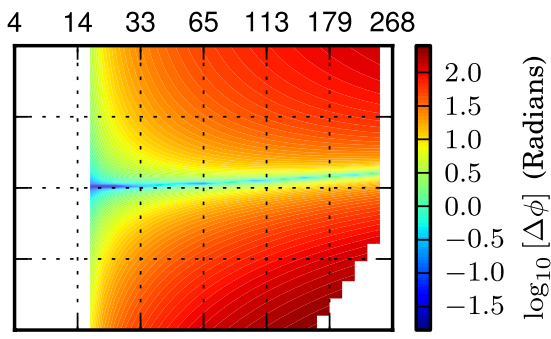

0.100 .150 .200 .250 .300 .350 .40

FIG. 9 (color online). The accumulation of phase differences between TaylorT2 and TaylorT4, for systems with component masses $\left(m_{1}, m_{2}\right)$ of $\left(1.4 M_{\odot}, 6 M_{\odot}\right)(\mathrm{left}),\left(1.4 M_{\odot}, 10 M_{\odot}\right)$ (center), and $\left(1.4 M_{\odot}, 14 M_{\odot}\right)$ (right). The approximants include spin terms up to 2.5PN. The calculation starts from the velocity corresponding to a gravitational-wave frequency of $15 \mathrm{~Hz}$, continues to the velocity on the horizontal axis, and reports the difference in accumulated gravitational-wave phase between the waveforms. The feature in the bottom right corner of each plot arises because the TaylorT2 approximant is no longer monotonic. Note that large phase differences accumulate at very low velocities $v \sim 0.2$ for even small black hole spins.

part of the discrepancy results from Gibbs phenomena differences between the approximants. It is important to note that neither of these waveforms have termination conditions that are determined by the physical behavior of the inspiralling binary. The termination frequency only indicates the point at which the approximant is certainly no longer valid. The increased match for aligned spin waveforms is due to the higher frequency cutoff, which pushes the termination frequency out of the most sensitive part of the zero-detuned, high-power aLIGO sensitivity curve.

Figure 8 shows a comparison between the TaylorR2F4 and TaylorT4 models. In that figure, the second expansion associated with the TaylorR2F4 model is extended to order $v^{9}$ (left) and $v^{12}$ (right), and the ending frequency of both is that corresponding to the MECO. We show that the TaylorR2F4 model is adequate for a large range of parameters as a computationally inexpensive substitute for TaylorT4.

Since the mismatch between the TaylorF2 and TaylorT4 models is not due to differences between the time domain and frequency domain approximants, this indicates that the effective higher order PN terms used in the construction of TaylorR2F4, which are also intrinsically present in TaylorT4, are still significant. To obtain better agreement between the different PN approximants we consider, it is necessary to extend the PN expansions of the energy and flux equations to include unknown higher order terms, particularly ones that involve the spin of the objects.

\section{ACCUMULATION OF PHASE DISCREPANCY}

In the previous sections, we demonstrated that the two PN approximants, TaylorF2 and TaylorT4, and the SEOBNRv1 model are not faithful to each other. We also showed that this is not due to the differences between frequency and time domain waveforms. From the construction of the TaylorR2F4 approximant, we also demonstrated that the two PN families can be written in a way that is consistent up to the chosen PN order, but where TaylorR2F4 contains higher order in $v$ corrections that
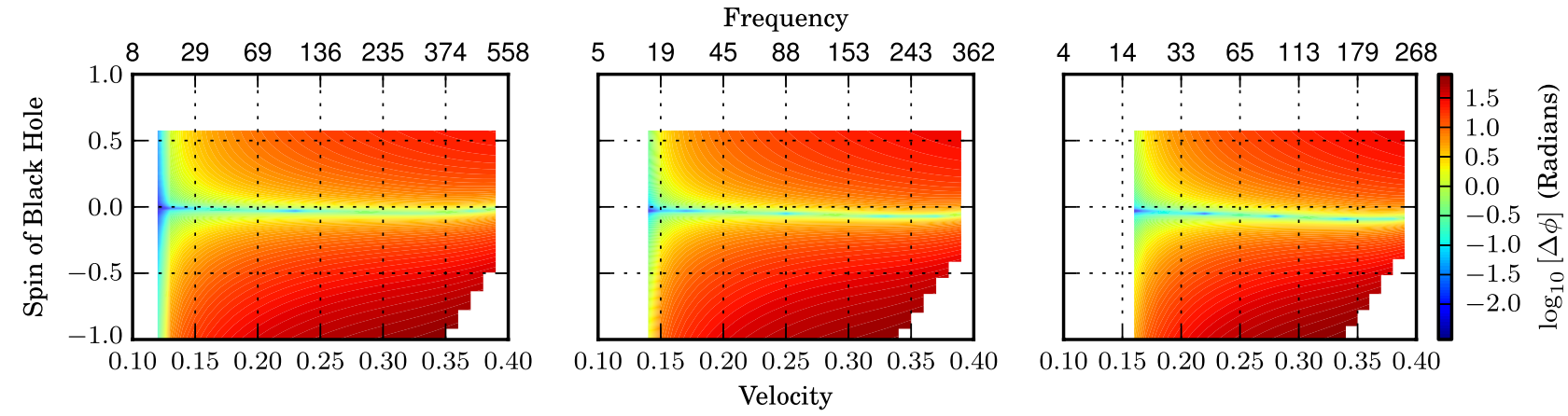

FIG. 10 (color online). The accumulation of phase difference between TaylorT2 and SEOBNRv1, for systems with component masses $\left(m_{1}, m_{2}\right)$ of $\left(6 M_{\odot}, 1.4 M_{\odot}\right)$ (left), $\left(10 M_{\odot}, 1.4 M_{\odot}\right)$ (center), and $\left(14 M_{\odot}, 1.4 M_{\odot}\right)$ (right). TaylorT2 includes spin terms up to 2.5PN. The calculation starts from the velocity corresponding to a gravitational-wave frequency of $15 \mathrm{~Hz}$, continues to the velocity on the horizontal axis, and reports the difference in accumulated gravitational-wave phase between the waveforms. The feature in the bottom right corner of each plot arises because the TaylorT2 approximant is no longer monotonic. As in Fig. 9, a large phase difference is accumulated at low velocities and small black hole spins. 

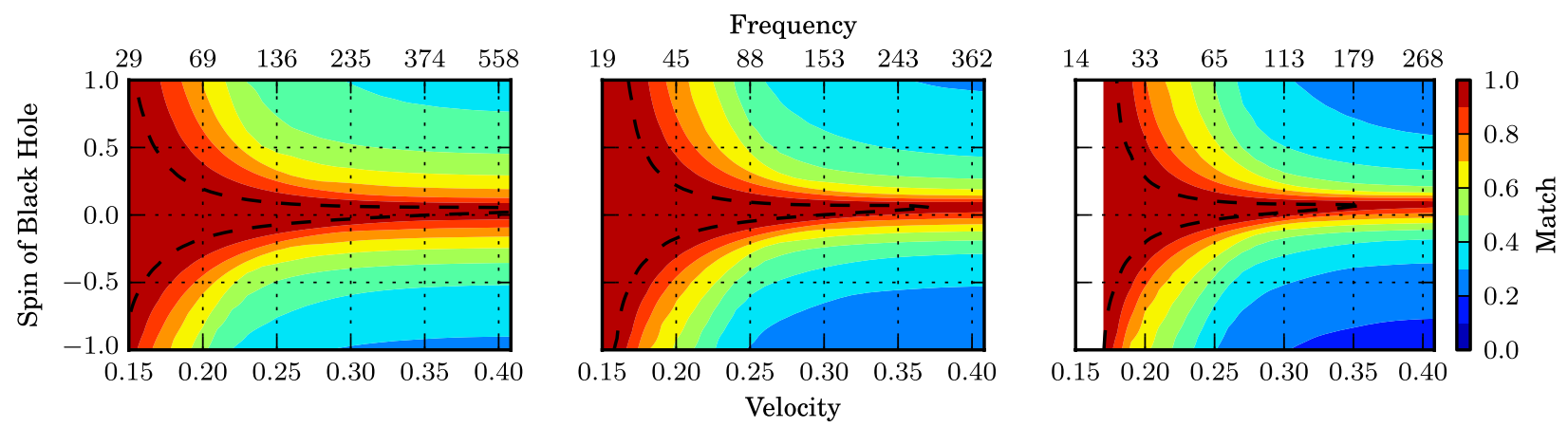

FIG. 11 (color online). The match between TaylorF2 and TaylorT4 integrated from $15 \mathrm{~Hz}$ up to the designated frequency for systems with component masses $\left(m_{1}, m_{2}\right)$ of $\left(1.4 M_{\odot}, 6 M_{\odot}\right)$ (left), $\left(1.4 M_{\odot}, 10 M_{\odot}\right)$ (center), and $\left(1.4 M_{\odot}, 14 M_{\odot}\right)$ (right). Both approximants include spin corrections up to 2.5PN. Matches are calculated using the aLIGO zero-detuned, high-power sensitivity curve. A contour at a match of 0.97 is indicated by the dotted line. The match follows the general features seen in the phase difference comparison of Fig. 9 and drops significantly, even at relatively low velocities. For the $\left(1.4 M_{\odot}, 6 M_{\odot}\right)$ system with a black hole spin $\chi=0.5$, the match has already dropped to $\sim 0.5$ at a velocity of only $\sim 0.25$.

account for the differences between the models. Since these are higher order corrections, they should start to become important to the orbital phasing only at high velocities, and thus high gravitational-wave frequencies. In this section we investigate where, for systems with parameters corresponding to NSBH binaries, the approximants diverge. We do this by examining the accumulation of phase as a function of orbital velocity and reporting the difference in the number of gravitational-wave cycles between different approximants.

In Fig. 9, we examine the difference in the accumulated phase between TaylorT2 and TaylorT4 for three example systems with component masses $\left(m_{1}, m_{2}\right)$ of $\left(6 M_{\odot}, 1.4 M_{\odot}\right)$, $\left(10 M_{\odot}, 1.4 M_{\odot}\right)$, and $\left(14 M_{\odot}, 1.4 M_{\odot}\right)$. We see that the phase difference between the two models quickly grows to tens of radians, even when the black hole spin magnitude is small. This is also true when comparing TaylorT2 and SEOBNRv1, as can be seen in Fig. 10. In the latter case, there is also a noticeable deviation away from the line of zero spin where for unknown reasons the two models diverge and subsequently converge.

\section{ACCUMULATION OF MISMATCH}

As gravitational-wave detectors are not directly sensitive to phase differences alone, it is useful to compute how the match, which incorporates the sensitivity of a gravitational-wave detector, changes as a function of the upper frequency cutoff used for the calculation. In this section we demonstrate at which frequencies and corresponding velocities the match between waveform families drops. To do so, we define an inner product between waveforms that is a function of the upper frequency cutoff. This inner product is then used in the match calculation of Eq. (19).

In Fig. 11, we examine the match between TaylorF2 and TaylorT4, integrated from a lower frequency cutoff of $15 \mathrm{~Hz}$ up to the upper frequency cutoff indicated on the horizontal axis. This is compared for the same three example systems as in Sec. VII. The match is shown across the range of allowable values of the black hole spin and the neutron star spin is set to zero. We see that the match drops precipitously even at low velocities and relatively
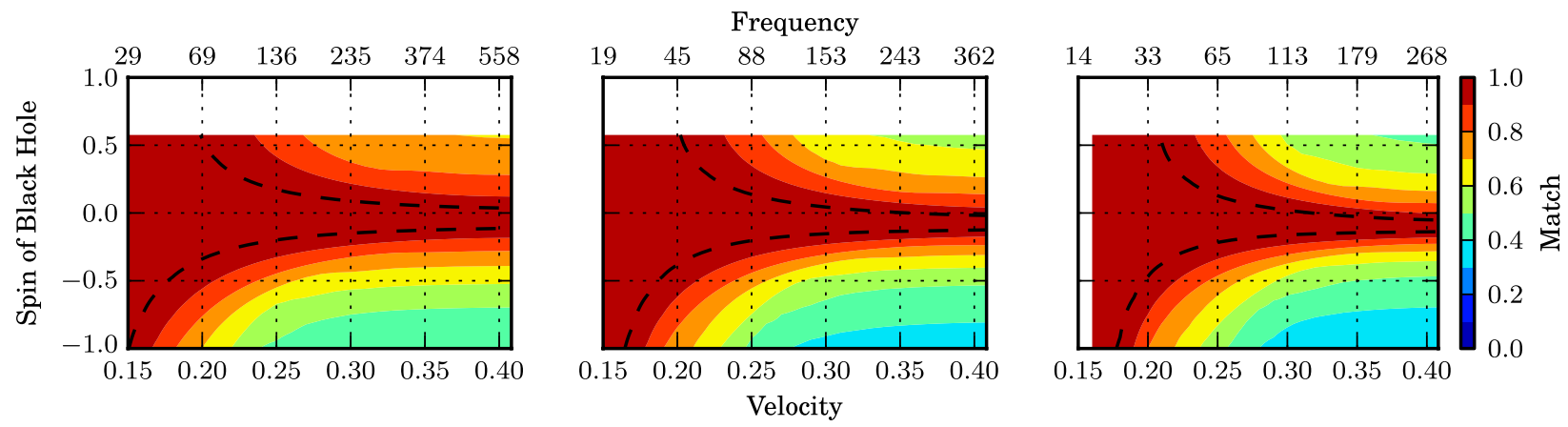

FIG. 12 (color online). The match between the TaylorF2 and SEOBNRv1 models integrated from $15 \mathrm{~Hz}$ up to the designated frequency for systems with component masses $\left(m_{1}, m_{2}\right)$ of $\left(6 M_{\odot}, 1.4 M_{\odot}\right)($ left $),\left(10 M_{\odot}, 1.4 M_{\odot}\right)($ center $)$, and $\left(14 M_{\odot}, 1.4 M_{\odot}\right)($ right $)$. TaylorF2 includes spin corrections up to 2.5PN. Matches are calculated using the aLIGO zero-detuned, high-power sensitivity curve. A contour at a match of 0.97 is indicated by the dotted line. We note that, although the match is marginally improved compared to Fig. 11, there are still large disagreements at velocities as low as 0.25 . 
modest spin magnitudes. For example, for a system with $m_{1}=6 M_{\odot}, m_{2}=1.4 M_{\odot}$, and a dimensionless spin of 0.5 for the black hole, the match drops below 0.7 at a velocity of only 0.23 . The loss in match is more pronounced with increasing mass ratio.

In Fig. 12, we examine the match between TaylorF2 and SEOBNRv1, integrated from a lower frequency cutoff of $15 \mathrm{~Hz}$ up to the upper frequency cutoff indicated on the horizontal axis. Again, the match drops for large spin magnitudes at relatively low velocities, although, just as the TaylorF2 approximant has shown better matches with the SEOBNRv1 approximant than with the TaylorT4 approximant, this occurs at somewhat higher velocities. This shows clearly that significant portions of the loss in match seen in Sec. IV occurs at unexpectedly low velocities.

\section{DETECTION SEARCHES AND EARLY ALIGO}

In the previous sections, we have demonstrated a substantial loss in match between different PN and EOB models of NSBH binaries. These discrepancies will cause substantial biases in attempts to measure the parameters of detected systems with aLIGO. However, when detecting systems the fitting factor, rather than the match, is the quantity that is used to assess the effectualness of a search [53]. The fitting factor maximizes the match between a signal and a bank of templates designed to capture e.g. $97 \%$ of the optimal signal-to-noise ratio. The template bank is constructed to be valid for the same range of masses and spins used throughout this paper and detailed in Sec I. Furthermore, the mass and spin parameters of these templates are strictly within this range. Discrepancies in match due to differing approximants may be compensated for by allowing a waveform to match to a template with shifted parameters. Figures 13 and 14 show the fitting factor of a TaylorF2 aligned spin template bank when used to detect aligned spin TaylorT4 waveforms. Figure 13 shows the distribution of fittings factors for approximants that include up to the 2.5PN spin corrections. Figure 14 demonstrates the effect of adding the higher order 3.0PN spin-orbit tail and 3.5PN spin-orbit corrections. Construction of these aligned spin banks use the method introduced in Ref. [14] and is described in more detail in Ref. [43].

There is substantial improvement in the fitting factors of aligned spin systems when adding the higher order spin corrections, but no improvement for antialigned spin systems. Although the loss in fitting factor is not as significant as the loss in match shown in Figs. 2 and 3, aLIGO NSBH searches will incur a significant loss in signal-to-noise ratio for systems with antialigned spins. Expanding the template bank may improve the fitting factors for particular systems. However, this would necessitate an increase in the size of the template bank, and subsequently cause an increase in the false alarm rate. If the faithfulness of NSBH waveforms is improved, this approach is no longer necessary.

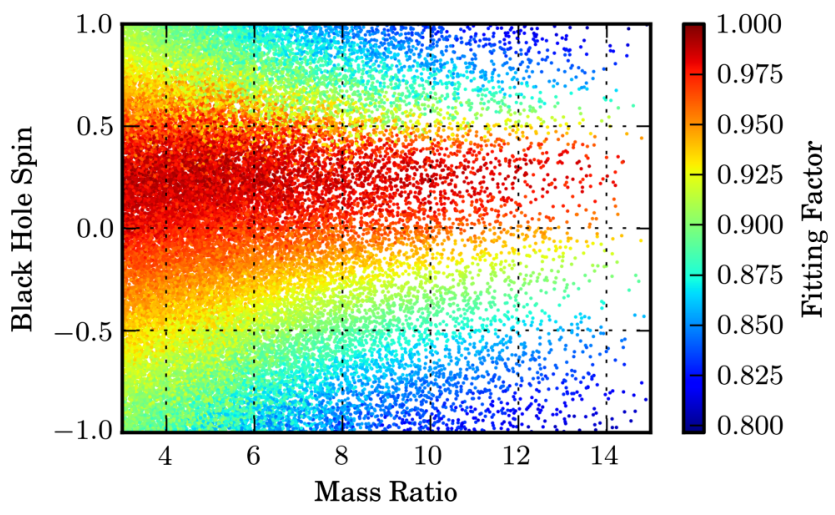

FIG. 13 (color online). The fitting factor between the TaylorF2 and TaylorT4 approximants as a function of the spin of the black hole and the mass ratio of the system, when maximizing the match over a bank of TaylorF2 waveforms. All approximants include spin corrections up to 2.5PN. Matches are calculated using the aLIGO zero-detuned, high-power sensitivity curve and a $15 \mathrm{~Hz}$ lower frequency cutoff. In comparison to the match of these approximants shown in Fig. 2, we see that while allowing for the maximization over a bank of templates has improved the overall agreement, it is unable to entirely make up for the poor match.

Refs. [13,50] suggest a 12-solar-mass cutoff for nonspinning, inpiral-only templates. While our figures consider a range of component masses, for a canonical neutron star of 1.4 solar masses, this roughly corresponds to a mass ratio of 8. Although the fitting factors in Figs. 13 and 14 are maximized over a parameter space that includes spin, we

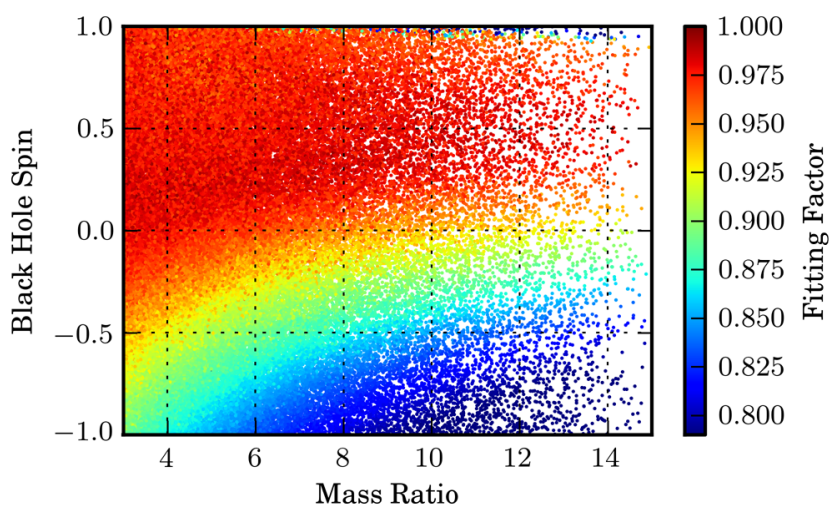

FIG. 14 (color online). The fitting factor between the TaylorF2 and TaylorT4 approximants as a function of the spin of the black hole and the mass ratio of the system, when maximizing the match over a bank of TaylorF2 waveforms. All approximants include the 3.5PN spin-orbit and 3.0PN spin-orbit tail corrections. Matches are calculated using the aLIGO zero-detuned, high-power sensitivity curve and a $15 \mathrm{~Hz}$ lower frequency cutoff. In comparison to the fitting factors shown in Fig. 13, we see that adding the higher order spin corrections has resulted in substantially improved fitting factors for systems where the spin is aligned with the orbital angular momentum. There is no improvement for antialigned systems. 


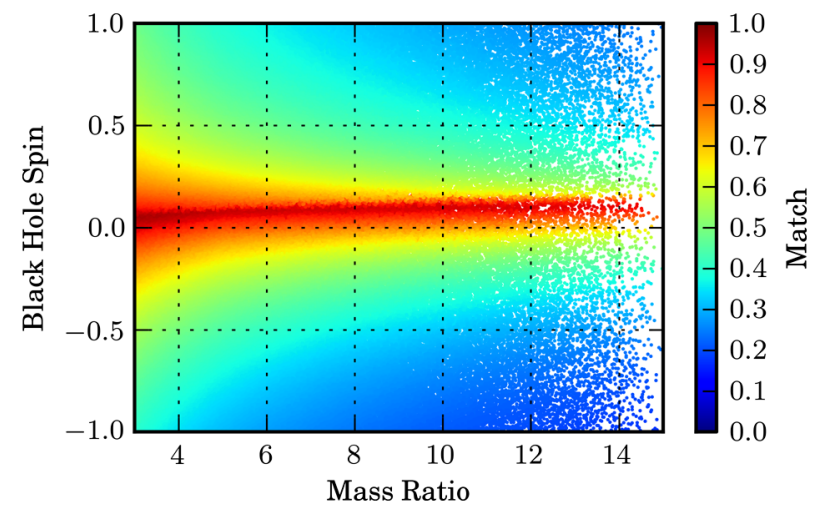

FIG. 15 (color online). The match between TaylorF2 and TaylorT4 as a function of the spin of the black hole and the mass ratio of the system. The approximants include spin corrections up to $2.5 \mathrm{PN}$. Matches are calculated using a $30 \mathrm{~Hz}$ lower frequency cutoff to approximate the sensitivity of an early aLIGO detector. In comparison to Fig. 2, which uses a $15 \mathrm{~Hz}$ lower frequency cutoff, there is only a negligible improvement in match. Matches remain low at moderate black hole spins $\chi \sim 0.3$.

find that where the spin of the black hole is nearly zero, the results are consistent with the comparisons of nonspinning approximants in Ref. [13].

In the previous sections we have modeled the sensitivity of aLIGO with the zero-detuned, high-power sensitivity curve [69]. Early commissioning scenarios for aLIGO indicate that observations will begin with less sensitivity in the 10-40 $\mathrm{Hz}$ region [52]. We investigate if the substantial disagreement found between TaylorF2 and TaylorT4 is still present for early detector sensitives by a instead using a lower frequency cutoff of $30 \mathrm{~Hz}$.

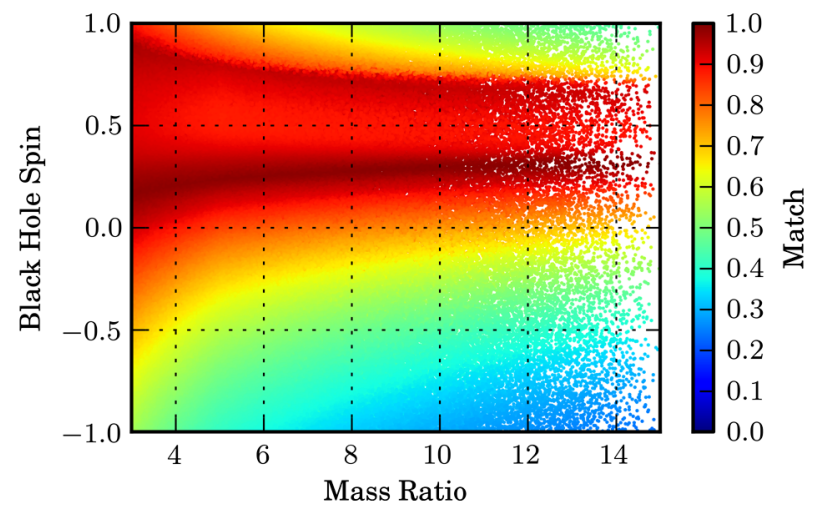

FIG. 16 (color online). The match TaylorF2 and TaylorT4 approximants, with the 3.5PN spin-orbit and 3.0PN spin-orbit tail corrections included, as a function of the spin of the black hole and the mass ratio of the system. The approximants include only the nown spin terms up to $2.5 \mathrm{PN}$. Matches are calculated using a $30 \mathrm{~Hz}$ lower frequency cutoff to approximate the sensitivity of the early aLIGO detector. In comparison to Fig. 3, which uses a $15 \mathrm{~Hz}$ lower frequency cutoff, there is only a negligible improvement in match.
In Fig. 15 and 16, we show the faithfulness between the TaylorF2 and TaylorT4 approximants that include only the complete 2.5 PN and partial 3.5PN spin-related corrections, respectively. We see that there is no significant improvement in the faithfulness of the approximants, and so additional spin corrections are desirable even for early detector scenarios.

\section{CONCLUSIONS}

We have found that there is significant disagreement between NSBH waveforms modeled with the TaylorT2, TaylorT4, and SEOBNRv1 approximants. This will pose problems for the construction of optimal NSBH detection searches, potentially reducing the event rate, and may cause significant biases in the parameter measurement of detected signals.

The discrepancies are not accounted for by the differences between frequency and time domain waveforms and start at fairly low $(v \sim 0.2)$ orbital velocities. Since the discrepancies in the approximants result from how the PN expansions of the energy and flux are combined and truncated, we conclude that the calculation of higher order PN terms is required to increase the faithfulness of these approximants, and more importantly, to improve the ability to detect NSBH coalescences. The discrepancies between approximants are significantly smaller when the spin of the black hole is close to zero, which further motivates the calculation of the PN terms associated with the spin of the objects beyond those known completely up to 2.5PN order and partially up to 3.5PN. Therefore, additional work is needed to verify the validity of waveform models used for NSBH searches. We also note that we have only compared different waveform families under the assumption that the spins of the component objects are (anti-)aligned with the orbital angular momentum of the system. It is expected that generic NSBH systems will not be limited to aligned spins, but may instead be more isotropically oriented. This could lead to an additional source of discrepancy between our models and the true signal, which would result in an additional loss in the detection rate of sources.

\section{ACKNOWLEDGMENTS}

We thank Stefan Ballmer, Alessandra Buonanno, Eliu Huerta, Prayush Kumar, Richard O'Shaughnessy, B. S. Sathyaprakash, Peter Saulson, and Matt West for useful discussions. This work is supported by National Science Foundation Awards No. PHY-0847611 (DAB, AHN), No. PHY-1205835 (AHN, IWH), No. PHY-0970074 (EO), and No. PHY-0855589 (AL). D. A. B., I. W. H., A.L., and E. O. thank the Kavli Institute for Theoretical Physics at Santa Barbara University, supported in part by NSF Grant No. PHY-0551164, for hospitality during this work. D. A.B. thanks the LIGO Laboratory Visitors Program, supported by NSF cooperative agreement 
No. PHY-0757058, for hospitality. D. K. and A. L. thank the Max Planck Gesellschaft for support. D. A. B. is supported by a Cottrell Scholar award from the Research Corporation for Science Advancement. Computations used in this work were performed on the Syracuse University Gravitation and Relativity cluster, which is supported by NSF Awards No. PHY-1040231 and No. PHY-1104371.

\section{APPENDIX A: POST-NEWTONIAN ENERGY AND GRAVITATIONAL-WAVE FLUX}

In this appendix, we give the $\mathrm{PN}$ coefficients for the center of mass energy $E_{i}$ and the gravitational-wave flux $F_{i}$, whose contributions were derived and presented in $[30,31,33,38-$ $40,70,73-77]$. We include corrections that involve the component objects' spins up to $3.5 \mathrm{PN}$. These coefficients depend on the dimensionless spins of the component objects $\chi_{i}=$ $\mathbf{S}_{i} / m_{i}^{2}$, their projections onto the direction of so-called Newtonian orbital angular momentum $\mathbf{L}_{N}=M \eta \mathbf{r} \times \dot{\mathbf{r}}$, and the symmetric mass ratio $\eta$. Additionally, quadrupolemonopole contributions depend on a parameter $q_{i}$, which quantifies the strength of the quadrupole moment induced by the oblateness of each spinning compact object. For BHs, $q_{i}=1$, while for NSs $q_{i}$ will depend on the equation of state, with [78] finding $q_{i} \sim 2-12$.

The coefficients associated with the energy are given as

$$
\begin{gathered}
E_{\text {Newt }}=-\frac{M}{2} \eta, \\
E_{2}=-\frac{3}{4}-\frac{1}{12} \eta, \\
E_{3}=\sum_{i=1}^{2}\left[\frac{8}{3}\left(\frac{m_{i}}{M}\right)^{2}+2 \eta\right]\left(\chi_{i} \cdot \hat{L}_{N}\right), \\
E_{4}=-\frac{27}{8}+\frac{19}{8} \eta-\frac{1}{24} \eta^{2} \\
+\eta\left[\chi_{1} \cdot \chi_{2}-3\left(\chi_{1} \cdot \hat{L}_{N}\right)\left(\chi_{2} \cdot \hat{L}_{N}\right)\right] \\
+\frac{1}{2} \sum_{i=1}^{2} q_{i}\left(\frac{m_{i}}{M}\right)^{2}\left[\chi_{i}^{2}-3\left(\chi_{i} \cdot \hat{L}_{N}\right)^{2}\right], \\
E_{5}=\sum_{i=1}^{2}\left[\left(8-\frac{31}{9} \eta\right)\left(\frac{m_{i}}{M}\right)^{2}+\eta\left(3-\frac{10}{3} \eta\right)\right]\left(\chi_{i} \cdot \hat{L}_{N}\right), \\
E_{6}=-\frac{675}{64}+\left(\frac{34445}{576}-\frac{205}{96} \pi^{2}\right) \eta-\frac{155}{96} \eta^{2}-\frac{35}{5184} \eta^{3}, \\
\left.+\eta\left(\frac{27}{4}-39 \eta+\frac{5}{4} \eta^{2}\right)\right]\left(\chi_{i} \cdot \hat{L}_{N}\right) . \\
E_{7}=\sum_{i=1}^{2}\left[\left(27-\frac{211}{4} \eta+\frac{7}{6} \eta^{2}\right)\left(\frac{m_{i}}{M}\right)^{2}\right. \\
\end{gathered}
$$

The coefficients associated with the flux are given as

$$
\begin{aligned}
& F_{\text {Newt }}=\frac{32}{5} \eta^{2} \\
& F_{2}=-\frac{1247}{336}-\frac{35}{12} \eta \\
& F_{3}=4 \pi-\sum_{i=1}^{2}\left[\frac{11}{4}\left(\frac{m_{i}}{M}\right)^{2}+\frac{5}{4} \eta\right]\left(\chi_{i} \cdot \hat{L}_{N}\right) \\
& F_{4}=-\frac{44711}{9072}+\frac{9271}{504} \eta+\frac{65}{18} \eta^{2} \\
& +\eta\left[\frac{289}{48}\left(\chi_{1} \cdot \hat{L}_{N}\right)\left(\chi_{2} \cdot \hat{L}_{N}\right)-\frac{103}{48} \chi_{1} \cdot \chi_{2}\right] \\
& +\sum_{i=1}^{2} q_{i}\left(\frac{m_{i}}{M}\right)^{2}\left[3\left(\chi_{i} \cdot \hat{L}_{N}\right)^{2}-\chi_{i}^{2}\right] \\
& +\frac{1}{96}\left(\frac{m_{i}}{M}\right)^{2}\left[7 \chi_{i}^{2}-\left(\chi_{i} \cdot \hat{L}_{N}\right)^{2}\right] \text {, } \\
& F_{5}=\left(-\frac{8191}{672}-\frac{583}{24} \eta\right) \pi+\sum_{i=1}^{2}\left[\left(-\frac{59}{16}+\frac{701}{36} \eta\right)\left(\frac{m_{i}}{M}\right)^{2}\right. \\
& \left.+\eta\left(-\frac{13}{16}+\frac{43}{4} \eta\right)\right]\left(\chi_{i} \cdot \hat{L}_{N}\right), \\
& F_{6}=\frac{6653739519}{69854400}+\frac{16}{3} \pi^{2}-\frac{1712}{105} \gamma_{E}-\frac{856}{105} \log \left(16 v^{2}\right) \\
& +\left(-\frac{134543}{7776}+\frac{41}{48} \pi^{2}\right) \eta-\frac{94403}{3024} \eta^{2}-\frac{775}{324} \eta^{3} \\
& -\pi \sum_{i=1}^{2}\left[\frac{65}{6}\left(\frac{m_{i}}{M}\right)^{2}-\frac{31}{6} \eta\right]\left(\chi_{i} \cdot \hat{L}_{N}\right), \\
& F_{7}=\left(-\frac{16285}{504}+\frac{214745}{1728} \eta+\frac{193385}{3024} \eta^{2}\right) \pi \\
& +\sum_{i=1}^{2}\left[\left(\frac{162035}{3888}+\frac{971}{54} \eta-\frac{6737}{108} \eta^{2}\right)\left(\frac{m_{i}}{M}\right)^{2}\right. \\
& \left.+\eta\left(\frac{9535}{336}+\frac{1849}{126} \eta-\frac{1501}{36} \eta^{2}\right)\right]\left(\chi_{i} \cdot \hat{L}_{N}\right) \text {. }
\end{aligned}
$$

\section{APPENDIX B: POST-NEWTONIAN APPROXIMANTS}

The PN approximants TaylorT4, TaylorT2, TaylorF2, and TaylorR2F4 are given using the flux up to $3.5 \mathrm{PN}$ and the center-of-mass energy up to 3.0 PN. Corrections due to spin are included up to $3.5 \mathrm{PN}$ order. This includes the leading order spin orbit correction $\beta$ at 1.5PN, leading order spin-spin correction $\sigma$ at $2 \mathrm{PN}$ (which includes quadrupole-monopole and so-called self-spin effects proportional to $s_{i}^{2}$ ), next-to-leading 
order spin-orbit corrections $\gamma$ at 2.5PN, tail-induced spin orbit correction $\xi$ at $3 \mathrm{PN}$, and third order spin-orbit correction $\zeta$ appearing at 3.5PN. These corrections can be expressed as

$$
\begin{array}{r}
\beta=\sum_{i=1}^{2}\left[\frac{113}{12}\left(\frac{m_{i}}{M}\right)^{2}+\frac{25}{4} \eta\right]\left(\chi_{i} \cdot \hat{L}_{N}\right), \\
\sigma=\eta\left[\frac{721}{48}\left(\chi_{1} \cdot \hat{L}_{N}\right)\left(\chi_{2} \cdot \hat{L}_{N}\right)-\frac{247}{48} \chi_{1} \cdot \chi_{2}\right] \\
+\frac{5}{2} \sum_{i=1}^{2} q_{i}\left(\frac{m_{i}}{M}\right)^{2}\left[3\left(\chi_{i} \cdot \hat{L}_{N}\right)^{2}-\chi_{i}^{2}\right] \\
+\frac{1}{96} \sum_{i=1}^{2}\left(\frac{m_{i}}{M}\right)^{2}\left[7 \chi_{i}^{2}-\left(\chi_{i} \cdot \hat{L}_{N}\right)^{2}\right],
\end{array}
$$

$$
\begin{aligned}
\gamma= & \sum_{i=1}^{2}\left[\left(\frac{732985}{2268}+\frac{140}{9} \eta\right)\left(\frac{m_{i}}{M}\right)^{2}\right. \\
& \left.+\eta\left(\frac{13915}{84}-\frac{10}{3} \eta\right)\right]\left(\chi_{i} \cdot \hat{L}_{N}\right)
\end{aligned}
$$

$$
\xi=\pi \sum_{i=1}^{2}\left[\frac{75}{2}\left(\frac{m_{i}}{M}\right)^{2}+\frac{151}{6} \eta\right]\left(\chi_{i} \cdot \hat{L}_{N}\right),
$$

$$
\begin{aligned}
\zeta= & \sum_{i=1}^{2}\left[\left(\frac{130325}{756}-\frac{796069}{2016} \eta+\frac{100019}{864} \eta^{2}\right)\left(\frac{m_{i}}{M}\right)^{2}\right. \\
& \left.+\eta\left(\frac{1195759}{18144}-\frac{257023}{1008} \eta+\frac{2903}{32} \eta^{2}\right)\right]\left(\chi_{i} \cdot \hat{L}_{N}\right) .
\end{aligned}
$$

\section{TaylorT4}

$$
\begin{aligned}
\frac{d v}{d t}= & \frac{32 \eta}{5 M} v^{9}\left\{1+\left(-\frac{743}{336}-\frac{11}{4} \eta\right) v^{2}+(4 \pi-\beta) v^{3}+\left(\frac{34103}{18144}+\frac{13661}{2016} \eta+\frac{59}{18} \eta^{2}+\sigma\right) v^{4}\right. \\
& +\left(-\frac{4159 \pi}{672}-\frac{189 \pi}{8} \eta-\frac{9}{40} \gamma+\left(\frac{743}{168}+\frac{11}{2} \eta\right) \beta\right) v^{5}+\left[\frac{16447322263}{139708800}-\frac{1712 \gamma_{E}}{105}+\frac{16 \pi^{2}}{3}-\frac{1712}{105} \log (4 v)\right. \\
& \left.\left.+\left(-\frac{56198689}{217728}+\frac{451 \pi^{2}}{48}\right) \eta+\frac{541}{896} \eta^{2}-\frac{5605}{2592} \eta^{3}-\xi\right] v^{6}+\pi\left(-\frac{4415}{4032}+\frac{358675}{6048} \eta+\frac{91495}{1512} \eta^{2}-\zeta\right) v^{7}\right\}
\end{aligned}
$$

\section{TaylorT2}

$$
\begin{aligned}
\frac{d t}{d v}= & \frac{5 M}{32 \eta} v^{-9}\left\{1+\left(\frac{743}{336}+\frac{11}{4} \eta\right) v^{2}+(-4 \pi+\beta) v^{3}+\left(\frac{3058673}{1016064}+\frac{5429}{1008} \eta+\frac{617}{144} \eta^{2}-\sigma\right) v^{4}\right. \\
& +\left(-\frac{7729 \pi}{672}+\frac{13 \pi}{8} \eta+\frac{9}{40} \gamma\right) v^{5}+\left[-\frac{10817850546611}{93884313600}+\frac{32 \pi^{2}}{3}+\frac{1712 \gamma_{E}}{105}+\frac{1712}{105} \log (4 v)\right. \\
& \left.+\left(\frac{3147553127}{12192768}-\frac{451 \pi^{2}}{48}\right) \eta-\frac{15211}{6912} \eta^{2}+\frac{25565}{5184} \eta^{3}-8 \pi \beta+\xi\right] v^{6} \\
& \left.+\pi\left(-\frac{15419335}{1016064}-\frac{75703}{6048} \eta+\frac{14809}{3024} \eta^{2}-\beta\left(\frac{8787977}{1016064}+\frac{51841}{2016} \eta+\frac{2033}{144} \eta^{2}\right)+\gamma\left(\frac{2229}{2240}+\frac{99}{80} \eta\right)+\zeta\right) v^{7}\right\}
\end{aligned}
$$

\section{TaylorF2}

$$
\begin{aligned}
A_{(F 2)}(f) \propto \frac{(\pi M f)^{2 / 3}}{\sqrt{\dot{F}(f)}} & \\
\psi_{(F 2)}(f)= & 2 \pi f t_{c}-\phi_{c}+\frac{3}{128 \eta} v^{-5}\left\{1+\left(\frac{3715}{756}+\frac{55}{9} \eta\right) v^{2}+(4 \beta-16 \pi) v^{3}+\left(\frac{15293365}{508032}+\frac{27145}{504} \eta+\frac{3085}{72} \eta^{2}-10 \sigma\right) v^{4}\right. \\
& +\left(\frac{38645}{756} \pi-\frac{65}{9} \pi \eta-\gamma\right)(1+3 \log (v)) v^{5}+\left[\frac{11583231236531}{4694215680}-\frac{640}{3} \pi^{2}-\frac{6848 \gamma_{E}}{21}-\frac{6848}{21} \log (4 v)\right. \\
& \left.+\left(-\frac{15737765635}{3048192}+\frac{2255 \pi^{2}}{12}\right) \eta+\frac{76055}{1728} \eta^{2}-\frac{127825}{1296} \eta^{3}+160 \pi \beta-20 \xi\right] v^{6}+\pi\left(\frac{77096675}{254016}+\frac{378515}{1512} \eta\right. \\
& \left.\left.-\frac{74045}{756} \eta^{2}+\beta\left(\frac{43939885}{254016}+\frac{259205}{504} \eta+\frac{10165}{36} \eta^{2}\right)-\gamma\left(\frac{2229}{112}-\frac{99}{4} \eta\right)-20 \zeta\right) v^{7}\right\}
\end{aligned}
$$




\section{TaylorR2F4}

In the equation below, the $a_{i}$ are the PN coefficients of the TaylorT4 expansion

$$
\left(\frac{d v}{d t}\right)_{T 4}=A_{7}(v)=a_{0}\left(1+\sum_{i=2}^{7} a_{i} v^{i}+a_{6 \log } v^{6} \log (4 v)\right),
$$

which can be read off of Eq. (B6),

$$
\begin{aligned}
\psi_{(R 2 F 4)}(f)= & \psi_{(F 2)}(f)+\frac{3}{128 \eta} v^{-5}\left\{\left[-20 \beta^{2}+\sigma\left(\frac{3715}{42}+100 \eta\right)\right] v^{6}+[(40 \beta-160 \pi) \sigma] v^{7}\right. \\
& +\frac{40}{9}\left[\left(3 a_{2}^{2} a_{4}-a_{2}^{4}-a_{4}^{2}-2 a_{3} a_{5}+3 a_{2} a_{3}^{2}-2 a_{2} a_{6}+\frac{2}{3} a_{2} a_{6 \log }\right)(1-3 \log (v))+3 a_{2} a_{6 \log } \log (4 v)^{2}\right] v^{8} \\
& +5\left[8 a_{2}^{2} a_{3}-2 a_{3}^{3}-12 a_{2} a_{3} a_{4}-6 a_{2}^{2} a_{5}+4 a_{4} a_{5}+4 a_{3} a_{6}-5 a_{3} a_{6 \log }+4 a_{2} a_{7}+4 a_{3} a_{6 \log } \log (4 v)\right] v^{9} \\
& +4\left[-a_{2}^{5}+6 a_{2}^{2} a_{3}^{2}+4 a_{2}^{3} a_{4}-3 a_{3}^{2} a_{4}-3 a_{2} a_{4}^{2}-6 a_{2} a_{3} a_{5}+a_{5}^{2}-3 a_{2}^{2} a_{6}+2 a_{4} a_{6}+2 a_{3} a_{7}\right. \\
& \left.+\left(\frac{21}{10} a_{2}^{2}-\frac{7}{5} a_{4}\right) a_{6 \log }+\left(2 a_{4} a_{6 \log }-3 a_{2}^{2} a_{6 \log }\right) \log (4 v)\right] v^{10}+\frac{20}{9}\left[-5 a_{2}^{4} a_{3}+4 a_{2} a_{3}^{3}+4 a_{2}^{3} a_{5}+12 a_{2}^{2} a_{3} a_{4}\right. \\
& -3 a_{3} a_{4}^{2}-3 a_{3}^{2} a_{5}-6 a_{2} a_{4} a_{5}-6 a_{2} a_{3} a_{6}+2 a_{5} a_{6}+3 a_{2} a_{3} a_{6 \log }-a_{5} a_{6 \log }+2 a_{4} a_{7}-3 a_{2}^{2} a_{7} \\
& \left.+\left(2 a_{5}-6 a_{2} a_{3}\right) a_{6 \log } \log (4 v)\right] v^{11}+\frac{10}{7}\left[a_{2}^{6}-10 a_{2}^{3} a_{3}^{2}+a_{3}^{4}-5 a_{2}^{4} a_{4}+12 a_{2} a_{3}^{2} a_{4}+6 a_{2}^{2} a_{4}^{2}-a_{4}^{3}+12 a_{2}^{2} a_{3} a_{5}\right. \\
& -6 a_{3} a_{4} a_{5}-3 a_{2} a_{5}^{2}+.4 a_{2}^{3} a_{6}-3 a_{3}^{2} a_{6}-6 a_{2} a_{4} a_{6}+a_{6}^{2}+\left(-\frac{11}{7} a_{2}^{3}+\frac{33}{28} a_{3}^{2}+\frac{33}{14} a_{2} a_{4}-\frac{11}{14} a_{6}+\frac{93}{392} a_{6 \log }\right) a_{6 \log } \\
& \left.\left.-6 a_{2} a_{3} a_{7}+2 a_{5} a_{7}+\left(4 a_{2}^{3}-3 a_{3}^{2}-6 a_{2} a_{4}+2 a_{6}-\frac{11}{14} a_{6 \log }\right) a_{6 \log } \log (4 v)+a_{6 \log }^{2} \log (4 v)^{2}\right] v^{12}\right\} .
\end{aligned}
$$

[1] G. M. Harry, Classical Quantum Gravity 27, 084006 (2010).

[2] F. Acernese et al., Virgo Technical Report No. 0027A-09, 2009.

[3] K.S. Thorne, in Three Hundred Years of Gravitation, edited by $\mathrm{S}$. Hawking and W. Israel (Cambridge University Press, Cambridge, England, 1987), p. 330.

[4] J. Abadie et al. (LIGO Scientific Collaboration, Virgo Collaboration), Classical Quantum Gravity 27, 173001 (2010).

[5] B. Sathyaprakash and B. F. Schutz, Living Rev. Relativity 12, 2 (2009).

[6] D. Eichler, M. Livio, T. Piran, and D. N. Schramm, Nature (London) 340, 126 (1989).

[7] R. Narayan, B. Paczynski, and T. Piran, Astrophys. J. 395, L83 (1992).

[8] B. Paczynski, Acta Astronaut. 41, 257 (1991).

[9] E. Berger, New Astron. Rev. 55, 1 (2011).

[10] C. L. Fryer, K. Belczynski, G. Wiktorowicz, M. Dominik, V. Kalogera, and D. E. Holz, Astrophys. J. 749, 91 (2012).

[11] M. Hannam, D. A. Brown, S. Fairhurst, C. L. Fryer, and I. W. Harry, Astrophys. J. 766, L14 (2013).

[12] L. Blanchet, Living Rev. Relativity 9, 4 (2006).

[13] A. Buonanno, B. R. Iyer, E. Ochsner, Y. Pan, and B. S. Sathyaprakash, Phys. Rev. D 80, 084043 (2009).
[14] D. A. Brown, I. Harry, A. Lundgren, and A. H. Nitz, Phys. Rev. D 86, 084017 (2012).

[15] B. Kiziltan, A. Kottas, and S.E. Thorsett, arXiv:1011.4291.

[16] P.C.C. Freire, S. M. Ransom, S. Bégin, I. H. Stairs, J. W. T. Hessels, L. H. Frey, and F. Camilo, Astrophys. J. 675, 670 (2008).

[17] K.-W. Lo and L.-M. Lin, Astrophys. J. 728, 12 (2011).

[18] J. W. Hessels, S. M. Ransom, I. H. Stairs, P. C. C. Freire, V. M. Kaspi et al., Science 311, 1901 (2006).

[19] F. Ozel, D. Psaltis, R. Narayan, and J.E. McClintock, Astrophys. J. 725, 1918 (2010).

[20] W. M. Farr, N. Sravan, A. Cantrell, L. Kreidberg, C. D. Bailyn, I. Mandel, and V. Kalogera, Astrophys. J. 741, 103 (2011).

[21] J. Miller, C. Reynolds, A. Fabian, G. Miniutti, and L. Gallo, Astrophys. J. 697, 900 (2009).

[22] P. C. Peters and J. Mathews, Phys. Rev. 131, 435 (1963).

[23] A. Wiseman, Phys. Rev. D 48, 4757 (1993).

[24] L. Blanchet, T. Damour, and B. R. Iyer, Phys. Rev. D 51, 5360 (1995).

[25] L. Blanchet, T. Damour, B. R. Iyer, C. M. Will, and A. Wiseman, Phys. Rev. Lett. 74, 3515 (1995).

[26] L. Blanchet, B. R. Iyer, C. M. Will, and A. G. Wiseman, Classical Quantum Gravity 13, 575 (1996). 
[27] L. Blanchet, G. Faye, B. R. Iyer, and B. Joguet, Phys. Rev. D 65, 061501 (2002).

[28] L. Blanchet, T. Damour, G. Esposito-Farese, and B. R. Iyer, Phys. Rev. Lett. 93, 091101 (2004).

[29] L. E. Kidder, C. M. Will, and A. G. Wiseman, Phys. Rev. D 47, R4183 (1993).

[30] L. E. Kidder, Phys. Rev. D 52, 821 (1995).

[31] K. G. Arun, A. Buonanno, G. Faye, and E. Ochsner, Phys. Rev. D 79, 104023 (2009).

[32] L. Blanchet, A. Buonanno, and G. Faye, arXiv:1210.0764.

[33] A. Bohe, S. Marsat, and L. Blanchet, Classical Quantum Gravity 30, 135009 (2013).

[34] L. E. Simone, S. W. Leonard, E. Poisson, and C. M. Will, Classical Quantum Gravity 14, 237 (1997).

[35] L. Blanchet, A. Buonanno, and G. Faye, Phys. Rev. D 84, 064041 (2011).

[36] S. Droz, D. J. Knapp, E. Poisson, and B. J. Owen, Phys. Rev. D 59, 124016 (1999).

[37] A. Buonanno, Y.-b. Chen, and M. Vallisneri, Phys. Rev. D 67, 104025 (2003).

[38] G. Faye, L. Blanchet, and A. Buonanno, Phys. Rev. D 74, 104033 (2006).

[39] L. Blanchet, A. Buonanno, and G. Faye, Phys. Rev. D 74, 104034 (2006).

[40] B. Mikoczi, M. Vasuth, and L. A. Gergely, Phys. Rev. D 71, 124043 (2005).

[41] E. Racine, A. Buonanno, and L. E. Kidder, Phys. Rev. D 80, 044010 (2009).

[42] D. A. Brown, A. Lundgren, and R. O'Shaughnessy, Phys. Rev. D 86, 064020 (2012).

[43] I. W. Harry, A. H. Nitz, D. A. Brown, A. Lundgren, E. Ochsner, and D. Keppel, arXiv:1307.3562 [Phys. Rev. D (to be published)].

[44] A. Taracchini, Y. Pan, A. Buonanno, E. Barausse, M. Boyle, T. Chu, G. Lovelace, H. P. Pfeiffer, and M. A. Scheel, Phys. Rev. D 86, 024011 (2012).

[45] M.D. Duez, Classical Quantum Gravity 27, 114002 (2010).

[46] M. Shibata and K. Taniguchi, Living Rev. Relativity 14, 6 (2011).

[47] F. Pannarale, arXiv:1208.5869.

[48] B. D. Lackey, K. Kyutoku, M. Shibata, P. R. Brady, and J. L. Friedman, arXiv:1303.6298.

[49] F. Foucart, L. Buchman, M. D. Duez, M. Grudich, and L. E. Kidder, Phys. Rev. D 88, 064017 (2013).

[50] D. A. Brown, P. Kumar, and A. H. Nitz, Phys. Rev. D 87, 082004 (2013).

[51] R. Smith, I. Mandel, and A. Vechhio, Phys. Rev. D 88, 044010 (2013).
[52] J. Aasi et al. (LIGO Scientific Collaboration, Virgo Collaboration), arXiv:1304.0670.

[53] T. A. Apostolatos, Phys. Rev. D 54, 2421 (1996).

[54] P. Peters, Phys. Rev. 136, B1224 (1964).

[55] B. Abbott et al. (LIGO Scientific Collaboration), Phys. Rev. D 69, 122001 (2004).

[56] B. Abbott et al. (LIGO Scientific Collaboration), Phys. Rev. D 72, 082002 (2005).

[57] B. Abbott et al. (LIGO Scientific Collaboration), Phys. Rev. D 72, 082001 (2005).

[58] B. Abbott et al. (LIGO Scientific Collaboration, TAMA Collaboration), Phys. Rev. D 73, 102002 (2006).

[59] B. Abbott et al. (LIGO Scientific Collaboration), Phys. Rev. D 77, 062002 (2008).

[60] B. P. Abbott et al. (LIGO Scientific Collaboration), Phys. Rev. D 80, 102001 (2009).

[61] B. P. Abbott et al. (LIGO Scientific Collaboration), Phys. Rev. D 80, 047101 (2009).

[62] J. Abadie et al. (LIGO and Virgo Scientific Collaborations), Phys. Rev. D 82, 102001 (2010).

[63] J. Abadie et al., Phys. Rev. D 85, 082002 (2012).

[64] B. Abbott et al. (LIGO Scientific Collaboration), Astrophys. J. 681, 1419 (2008).

[65] J. Abadie et al. (LIGO and Virgo Scientific Collaborations), Astrophys. J. 715, 1453 (2010).

[66] M. Briggs et al. (LIGO Scientific Collaboration), Astrophys. J. 760, 12 (2012).

[67] L. A. Wainstein and V. D.Zubakov, Extraction of Signals from Noise (Prentice-Hall, Englewood Cliffs, New Jersey, 1962).

[68] B. Allen, W. G. Anderson, P. R. Brady, D. A. Brown, and J. D. Creighton, Phys. Rev. D 85, 122006 (2012).

[69] B. Abbott et al. (LIGO Scientific Collaboration), Tech. Rep. No. LIGO-T0900288-v3, 2010.

[70] A. Bohe, S. Marsat, G. Faye, and L. Blanchet, Classical Quantum Gravity 30, 075017 (2013).

[71] T. Damour, B. R. Iyer, and B. S. Sathyaprakash, Phys. Rev. D 63, 044023 (2001).

[72] J. Aasi et al. (LIGO Collaboration, Virgo Collaboration), arXiv:1304.1775.

[73] T. Damour, P. Jaranowski, and G. Schaefer, Phys. Rev. D 62, 044024 (2000).

[74] L. Blanchet and G. Faye, Phys. Lett. A 271, 58 (2000).

[75] L. Blanchet, B. R. Iyer, and B. Joguet, Phys. Rev. D 65, 064005 (2002).

[76] E. Poisson, Phys. Rev. D 57, 5287 (1998).

[77] S. Marsat, A. Bohe, G. Faye, and L. Blanchet, Classical Quantum Gravity 30, 055007 (2013).

[78] W. G. Laarakkers and E. Poisson, Astrophys. J. 512, 282 (1999). 